\title{
Cosmic Web of Galaxies in the COSMOS Field: Public Catalog and Different Quenching for Centrals and Satellites
}

\author{
Behnam Darvish $^{1}$, Bahram Mobasher ${ }^{2}$, D. Christopher Martin ${ }^{1}$, David Sobral ${ }^{3,4}$, Nick Scoville ${ }^{1}$, Andra Stroe $^{5}$, \\ Shoubaneh Hemmati ${ }^{6}$, and Jeyhan Kartaltepe ${ }^{7}$ \\ ${ }^{1}$ Cahill Center for Astrophysics, California Institute of Technology, 1216 East California Boulevard, Pasadena, CA 91125, USA; \\ bdarv@caltech.edu, bdarv001@ucr.edu \\ ${ }^{2}$ University of California, Riverside, 900 University Avenue, Riverside, CA 92521, USA \\ ${ }^{3}$ Department of Physics, Lancaster University, Lancaster, LA1 4 YB, UK \\ ${ }^{4}$ Leiden Observatory, Leiden University, P.O. Box 9513, NL-2300 RA Leiden, The Netherlands \\ ${ }^{5}$ European Southern Observatory, Karl-Schwarzschild-Str. 2, D-85748, Garching, Germany \\ ${ }^{6}$ Infrared Processing and Analysis Center, California Institute of Technology, Pasadena, CA 91125, USA \\ ${ }^{7}$ School of Physics and Astronomy, Rochester Institute of Technology, 84 Lomb Memorial Drive, Rochester, NY 14623, USA \\ Received 2016 November 3; revised 2016 December 23; accepted 2017 January 5; published 2017 February 28
}

\begin{abstract}
We use a mass complete $\left(\log \left(M / M_{\odot}\right) \geqslant 9.6\right)$ sample of galaxies with accurate photometric redshifts in the COSMOS field to construct the density field and the cosmic web to $z=1.2$. The comic web extraction relies on the density field Hessian matrix and breaks the density field into clusters, filaments, and the field. We provide the density field and cosmic web measures to the community. We show that at $z \lesssim 0.8$, the median star formation rate (SFR) in the cosmic web gradually declines from the field to clusters and this decline is especially sharp for satellites $(\sim 1$ dex versus $\sim 0.5$ dex for centrals). However, at $z \gtrsim 0.8$, the trend flattens out for the overall galaxy population and satellites. For star-forming (SF) galaxies only, the median SFR is constant at $z \gtrsim 0.5$ but declines by $\sim 0.3-0.4$ dex from the field to clusters for satellites and centrals at $z \lesssim 0.5$. We argue that for satellites, the main role of the cosmic web environment is to control their SF fraction, whereas for centrals, it is mainly to control their overall SFR at $z \lesssim 0.5$ and to set their fraction at $z \gtrsim 0.5$. We suggest that most satellites experience a rapid quenching mechanism as they fall from the field into clusters through filaments, whereas centrals mostly undergo a slow environmental quenching at $z \lesssim 0.5$ and a fast mechanism at higher redshifts. Our preliminary results highlight the importance of the large-scale cosmic web on galaxy evolution.
\end{abstract}

Key words: galaxies: evolution - galaxies: high-redshift - large-scale structure of universe

Supporting material: machine-readable table

\section{Introduction}

The standard model of cosmology is based on the cosmological principle, the concept of a spatially homogeneous and isotropic universe when averaged over scales of $\gtrsim 100 \mathrm{Mpc}$. On smaller scales, the universe is inhomogeneous. Dark matter, gas, and galaxies are organized in a complex network known as the cosmic web (Bond et al. 1996), which is a direct consequence of the anisotropic gravitational collapse of matter from the early seeds of primordial matter fluctuations (Zel'dovich 1970). The cosmic web has a broad dynamical range of environments over different physical scales and densities: voids that are deprived of matter and occupy much of the volume of the web, planar walls and sheets, filamentary structures that form at the intersection of walls, and dense clusters and groups of galaxies woven together by filaments. This large-scale picture of the universe has been revealed in numerical simulations and observed distribution of galaxies in the local universe (Davis et al. 1985; Geller \& Huchra 1989; Bond et al. 1996; Colless et al. 2003; Doroshkevich et al. 2004; Jarrett 2004; Jones et al. 2009; Alpaslan et al. 2014a). Galaxies form and evolve in the cosmic web and their evolution should be essentially driven by a combination of internal and external processes.

Filaments make the backbone of the cosmic web, comprising $\sim 40 \%$ of the total mass in the local universe (Aragón-Calvo et al. 2010b), presumably containing a large fraction of missing baryons in the form of a warm-hot intergalactic medium (IGM) gas (Briel \& Henry 1995; Cen \& Ostriker 1999; Scharf et al. 2000; Davé et al. 2001; Zappacosta et al. 2002; Nicastro et al. 2005; Shull et al. 2012; Haider et al. 2016) and potentially hosting much of the star formation activity in the universe (Snedden et al. 2016). Recent models of galaxy formation heavily rely on the cold gas flow into galaxies through streams of filaments (Kereš et al. 2005; Dekel et al. 2009), with recent observational evidence supporting this picture (Cantalupo et al. 2014; Martin et al. 2014a, 2014b, 2015, 2016). The absorption of photons passing through the IGM of the cosmic web has been used to constrain the properties of the IGM and to shed light on the physics and nature of reionization (e.g., see the review by Becker et al. 2015). The cosmic web is currently used to significantly improve the photometric redshift accuracy of surveys (e.g., Aragón-Calvo et al. 2015). The structure, properties, and evolution of the cosmic web contain a wealth of information about the initial matter distribution in the universe with valuable cosmological implications (e.g., see Wang et al. 2016 and the references therein).

Therefore, it is of great importance to characterize and describe the cosmic web of galaxies. However, the multi-scale nature of the cosmic web, its complexity and connectivity, and the lack of a fully objective method in identifying its major components make such studies challenging. Nonetheless, several methods have been developed to quantify and extract the components of the cosmic web in both simulations and observational data (e.g., see Cautun et al. 2014 for a review). 
Some of these methods are designed to specifically extract certain components of the web, for example, only filaments (Pimbblet 2005; Stoica et al. 2005, 2010; Novikov et al. 2006; Sousbie et al. 2008; Bond et al. 2010; González \& Padilla 2010; Smith et al. 2012; Tempel et al. 2014), and some are able to simultaneously break the cosmic web into its major components (e.g., Aragón-Calvo et al. 2007a, 2010a; Colberg 2007; Hahn et al. 2007b; Forero-Romero et al. 2009; Jasche et al. 2010; Sousbie 2011; Falck et al. 2012; Hoffman et al. 2012; Wang et al. 2012; Leclercq et al. 2015; Snedden et al. 2015). We particularly mention those that take the multiscale nature of the cosmic web into account, such as the Multiscale Morphology Filter (MMF) algorithm (Aragón-Calvo et al. 2007a; also see Cautun et al. 2013).

These methods have been mostly applied to simulations and some observational data sets with quite interesting results. For example, trends between the dependence of spin, shape, size, and other properties of halos and galaxies on the cosmic web and orientation of filaments and walls are found in simulations (Altay et al. 2006; Aragón-Calvo et al. 2007b; Hahn et al. 2007a, 2007b; Zhang et al. 2009; Codis et al. 2012; Libeskind et al. 2013; Trowland et al. 2013; Dubois et al. 2014; Chen et al. 2015, 2016a; Kang \& Wang 2015; Welker et al. 2015; Gonzalez et al. 2016) and observations (Kashikawa \& Okamura 1992; Navarro et al. 2004; Lee \& Erdogdu 2007; Paz et al. 2008; Jones et al. 2010; Tempel \& Libeskind 2013; Tempel et al. 2013; Zhang et al. 2013), generally in support of the Tidal Torque Theory (Peebles 1969; White 1984; Codis et al. 2015) as our comprehension of the origin of the spin of galaxies (also see Kiessling et al. 2015; Joachimi et al. 2015 for reviews).

Of great interest is the quenching of galaxies in the cosmic web. Generally, two major quenching mechanisms are proposed, the "environmental quenching" and "mass quenching." The later is thought to be associated with, e.g., active galactic nuclei and stellar feedback (e.g., Fabian 2012; Hopkins et al. 2014). The environmental quenching processes such as ram pressure stripping (e.g., Gunn \& Gott 1972; Abadi et al. 1999), galaxygalaxy interactions and harassment (e.g., Farouki \& Shapiro 1981; Merritt 1983; Moore et al. 1998), and strangulation (e.g., Larson et al. 1980; Balogh et al. 2000) act in medium to high density environments, with different quenching timescales. These processes seem to depend on galaxy properties as well. For example, ram pressure stripping is more effective on lessmassive galaxies as these systems have weaker binding gravitational potential. Mass quenching has been mostly attributed to central galaxies, whereas environmental quenching is primarily linked to satellites (e.g., Peng et al. 2012; Kovač et al. 2014). Moreover, environmental and mass quenching processes seem to suppress star formation activity independent of each other (e.g., Peng et al. 2010; Quadri et al. 2012), although this has been questioned recently. For example, Darvish et al. (2016) showed that environmental quenching is more efficient for more massive galaxies and mass quenching is more efficient in denser environments. Interestingly, AragónCalvo et al. (2016) recently showed that the stripping of the filamentary web around galaxies is responsible for star formation quenching, without the need for feedback processes.

However, it is still not fully clear whether the environmental effects on galaxy quenching are a local phenomenon or also act on global large-scale cosmic web environments as well. For example, the "galactic conformity"- the observation that satellites are more likely to be quenched around quiescent centrals than star-forming (SF) ones-has been found on both small and larger megaparsec scales (Weinmann et al. 2006; Kauffmann et al. 2013; Hartley et al. 2015; Hearin et al. 2015; Berti et al. 2017; Hatfield \& Jarvis 2016; Kawinwanichakij et al. 2016), suggesting the role of the large-scale gravitational tidal field on galaxy properties. Moreover, several observations have found that the star formation activity and other galaxy properties depend on the large-scale cosmic web (Fadda et al. 2008; Porter et al. 2008; Biviano et al. 2011; Darvish et al. 2014; Ricciardelli et al. 2014; Chen et al. 2016b; Darvish et al. 2015a; Guo et al. 2015; Alpaslan et al. 2016; Pandey \& Sarkar 2016), whereas others have seen no or at best a weak dependence between the properties of galaxies and the global cosmic web environments (Alpaslan et al. 2015; Eardley et al. 2015; Filho et al. 2015; Penny et al. 2015; Alonso et al. 2016; Beygu et al. 2016; Brouwer et al. 2016; Vulcani et al. 2016a).

Nonetheless, the majority of these cosmic web studies are limited to numerical simulations or large spectroscopic surveys in the local universe such as SDSS and GAMA (e.g., Alpaslan et al. 2014a; Tempel et al. 2014), mainly due to the completeness, selection function, and projection effect issues involved in observations. Using spectroscopic samples has the benefit of constructing the density field in three-dimensions (3D), which suffers less from projection effects. Moreover, establishing the vectorial properties of the cosmic web, for example, the direction of filaments in 3D is possible. However, redshift-space distortions such as the finger-of-god effect should be carefully taken into account so that components of the cosmic web would not be misclassified (e.g., filaments versus finger-of-god elongated clusters).

Currently, there are not enough spectroscopic redshifts available at higher redshifts to perform similar studies. To extend the cosmic web studies to higher redshifts, one could alternatively use photometric information in two-dimensional (2D) redshift slices, as long as the uncertainties in the photometric redshifts are not too large. The information contained in 3D vectorial properties of the cosmic web is usually lost in 2D analyses. However, the scalar quantities such as star formation rate (SFR) and stellar mass of galaxies in the cosmic web, on average, and in a statistical sense, are still measurable in $2 \mathrm{D}$ projections. The higher redshift studies of the cosmic web are particularly important as its components are not fully evolved and gravitationally merged yet, and much information regarding the properties of galaxies and dark matter halos, that would otherwise get lost due to the non-linearinteraction regime, is still maintained (e.g., see Jones et al. 2010; Cautun et al. 2014). This sets the need for contiguous largevolume surveys at higher redshifts, with negligible cosmic variance, that are equipped with very accurate photometric redshifts to high- $z$. The COSMOS field survey (Scoville et al. $2007 b$ ) is ideal for such cosmic web studies to higher redshifts.

In pilot studies to target the cosmic web, Darvish et al. (2014, 2015a) used the 2D version of the MMF algorithm and applied it to potential large-scale structures in the COSMOS at $z \sim 0.83$ and 0.53 . The $z \sim 0.83$ structure clearly showed a filament linking several clusters and groups and was traced by the distribution of $\mathrm{H} \alpha$ emitters (Sobral et al. 2011; Darvish et al. 2014). Further studies of the structure showed that although stellar mass, SFR, and the main sequence of SF galaxies are invariant to the cosmic web, the fraction of $\mathrm{H} \alpha$ emitters is enhanced in filaments, likely due to galaxy-galaxy interactions. The other potential filament at $z \sim 0.53$ was spectroscopically 
confirmed and the spectroscopic analysis also showed that although many properties of SF galaxies, such as stellar-todynamical mass ratio and ionization parameters, are independent of their cosmic web environment, gas-phase metallicities are slightly higher in filaments relative to the field and electron densities are significantly lower (Darvish et al. 2015a). These are properties shared with SF galaxies found in merging clusters, potentially suggesting a connection (Sobral et al. 2015).

These single-structure studies show the potential role of the cosmic web on galaxy evolution. However, small sample size is one of the major issues in these studies. The robustness of our cosmic web detection algorithm in revealing the large-scale cosmic web, the need for a large homogeneously selected sample of galaxies located in different regions of the web and extended to higher $\mathrm{z}$, and the limited number of studies that consider the explicit role of the comic web on galaxy evolution, motivate us to extend our analysis to a reliably large sample of galaxies in the whole COSMOS field to $z \sim 1.2$. Therefore, the focus of this paper is to provide a catalog of density field of galaxies, cosmic web components, and their galactic content over a large and reliable redshift range to the community. We also investigate the star formation activity of central and satellite galaxies in the global cosmic web environments.

The format of this paper is as follows. In Section 2, we briefly review the data. Section 3 outlines the methods used to determine the density field, the comic web extraction, galaxy classification, and the SFR and stellar mass estimation for our sample. In Section 4, we present the main results and discus$\mathrm{s}$ and compare them with the literature. A summary of this work is given in Section 5.

Throughout this work, we assume a flat $\Lambda$ CDM cosmology with $H_{0}=70 \mathrm{kms}^{-1} \mathrm{Mpc}^{-1}, \Omega_{m}=0.3$, and $\Omega_{\Lambda}=0.7$. All magnitudes are in the $\mathrm{AB}$ system and SFRs and stellar masses are based on a Chabrier (Chabrier 2003) initial mass function (IMF).

\section{Data and Sample Selection}

In this work, we use the $\sim 1.8 \mathrm{deg}^{2}$ COSMOS field (Capak et al. 2007; Scoville et al. 2007b), which is ideal for the largescale structure studies at $z \gtrsim 0.1$, with minimal cosmic variance and a wealth of ancillary data. Using the Moster et al. (2011) recipe, the cosmic variance even for the most massive galaxies $\left(\log \left(M / M_{\odot}\right)>11\right)$ in this field is only $\sim 15 \%-10 \%$ at $z \sim 0.1-3$.

Here, we use the latest COSMOS2015 photometric redshift (photo-z) catalog (Laigle et al. 2016) in the UltraVISTA-DR2 region (McCracken et al. 2012; Ilbert et al. 2013). This comprises ground- and space-based photometric data in more than 30 bands (Section 3.1). We select objects that are flagged as galaxies, located in the range $149.33<\alpha_{2000}(\mathrm{deg})<150.8$ and $1.6<\delta_{2000}(\mathrm{deg})<2.83$, and are in the redshift range $0.1<z<1.2$. following our discussion in Section 3.1, we limit our study to $0.1<z<1.2$ to guarantee a reliable density field and cosmic web estimation using very accurate photo-zs $\left(\sigma_{\Delta z /(1+z)} \lesssim 0.01\right)$.

In addition to the aforementioned criteria, we apply a cut based on the stellar mass completeness of the survey (Section 3.2). All galaxies more massive than the mass completeness limit of the highest redshift of this study at $z=1.2$ are selected $\left(\log \left(M / M_{\odot}\right) \geqslant 9.6\right.$; Section 3.2). This is equivalent to a volume-limited sample. We use this sample to estimate the density field (Section 3.3), to extract the cosmic

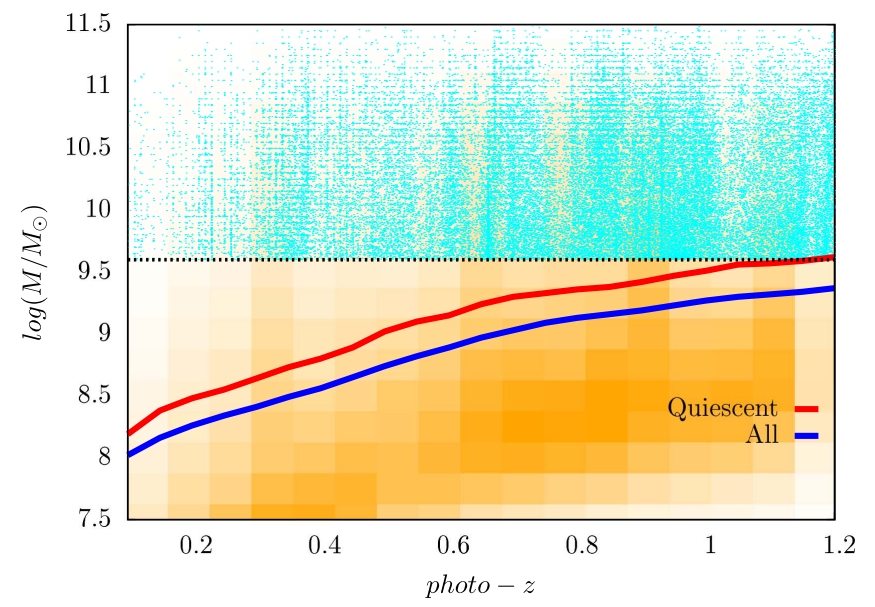

Figure 1. Stellar mass of galaxies as a function of redshift, shown as an orange heat map. The blue and red lines show the estimated stellar mass completeness limit for all the galaxies (star-forming and quiescent) and quiescent galaxies only, respectively. At each redshift, we define the mass completeness limit as the stellar mass for which $90 \%$ of galaxies have their limiting mass below it (Section 3.2). All galaxies that are more massive than the mass completeness limit of quiescent galaxies for the highest redshift of this study at $z=1.2$ are selected $\left(\log \left(M / M_{\odot}\right)\right.$ $\geqslant 9.6$ ) for our analysis (cyan points). This is similar to a volume-limited selection.

web components (Section 3.4), and to conduct the analysis in Section 4. Figure 1 shows the mass completeness limit and the galaxies selected in this study.

For the analysis here, we only rely on galaxies that are not close to the edge of the field and large masked areas, as the density values and cosmic web assignment for galaxies close to these regions are not reliable. The total number of galaxies before (and after) discarding those near the edge and masked regions is 45421 (38865), respectively. We flag galaxies located near the edge or masked areas in Table 1.

\section{Methods}

\subsection{Photo-z Accuracy}

In this study, we use the photo- $z$ of galaxies to construct the density field and extract the cosmic web components. Using photometric redshifts automatically suppresses the redshiftspace distortions such as the finger-of-god effect. However, large photometric redshift uncertainties would erode and smooth out the real structures in the density field, especially in the densest regions.

A number of studies have shown that using photo-zs with typical uncertainties of $\sigma_{\Delta z /(1+z)} \lesssim 0.01$ can still fairly accurately construct the density field (e.g., Cooper et al. 2005; Malavasi et al. 2016), with more optimistic studies such as Lai et al. (2016) showing that even larger uncertainties can still reveal the general environmentally driven trends. Therefore, reliable and accurate photometric redshift measurements are of crucial importance.

Here, we use the photometric redshifts from the COSMOS2015 catalog (Laigle et al. 2016), which are estimated using over 30 bands from near-UV to far-IR wavelengths. A comparison with the zCOSMOS bright spectroscopic redshift sample (Lilly et al. 2009) to $z \sim 1$ shows that photo- $z$ accuracy is $\sigma_{\Delta z /\left(1+z_{s}\right)} \sim 0.007$, with a catastrophic failure fraction of only $\sim 0.5 \%$ (Laigle et al. 2016). Figure 2 shows the photo- $z$ uncertainties, $\sigma_{\Delta z /(1+z)}$, as a function of redshift for our sample, along with the median photo- $z$ uncertainties (red line). Median uncertainties are estimated within \pm 0.2 redshift intervals at 
Table 1

Sample Galaxies with Estimated Density Values, Cosmic Web Environments, and Galaxy Type

\begin{tabular}{|c|c|c|c|c|c|c|c|c|c|c|c|}
\hline $\begin{array}{l}\alpha_{2000} \\
(\mathrm{deg})\end{array}$ & $\begin{array}{l}\delta_{2000} \\
(\mathrm{deg})\end{array}$ & Photo-z & $\begin{array}{l}\text { Density } \\
\left(\mathrm{Mpc}^{-2}\right)\end{array}$ & Overdensity & $\begin{array}{l}\text { Cluster } \\
\text { Signal }\end{array}$ & $\begin{array}{l}\text { Filament } \\
\text { Signal }\end{array}$ & $\begin{array}{l}\text { Cosmic Web } \\
\text { Environment }\end{array}$ & Group ID & $\begin{array}{c}\text { Number of } \\
\text { Group Members }\end{array}$ & $\begin{array}{l}\text { Galaxy } \\
\text { Type }\end{array}$ & Flag $^{\mathrm{a}}$ \\
\hline 150.041038 & 1.679104 & 0.2200 & 2.17 & 0.78 & 0.000411 & 0.236093 & filament & 74 & 3 & central & 0 \\
\hline 149.468224 & 1.660186 & 0.6036 & 3.38 & 1.45 & 0.050772 & 0.062457 & filament & 1656 & 5 & central & 0 \\
\hline 149.854923 & 1.661894 & 0.2611 & 0.33 & 0.12 & 0.000004 & 0.002267 & field & -99 & -99 & isolated & 0 \\
\hline 149.849106 & 1.660836 & 0.7437 & 1.96 & 0.78 & 0.133678 & 0.218654 & filament & 2602 & 14 & satellite & 0 \\
\hline 149.570287 & 1.660729 & 0.6966 & 1.01 & 0.41 & 0.001817 & 0.010613 & field & -99 & -99 & isolated & 0 \\
\hline 149.431513 & 1.660398 & 0.9849 & 0.29 & 0.12 & 0.000000 & 0.001720 & field & 5302 & 2 & central & 0 \\
\hline 149.734774 & 1.660589 & 0.7550 & 0.77 & 0.30 & 0.000413 & 0.000150 & field & -99 & -99 & isolated & 0 \\
\hline 150.261428 & 1.660046 & 0.9069 & 7.34 & 3.05 & 0.161472 & 0.155610 & field & 4043 & 5 & satellite & 0 \\
\hline 149.596887 & 1.660786 & 0.8669 & 0.47 & 0.19 & 0.000309 & 0.006520 & field & 3845 & 3 & central & 0 \\
\hline 149.942127 & 1.660848 & 1.0543 & 2.12 & 0.99 & 0.057414 & 0.114930 & filament & -99 & -99 & isolated & 0 \\
\hline 149.778184 & 1.660923 & 0.9624 & 1.14 & 0.49 & 0.085444 & 0.056621 & field & -99 & -99 & isolated & 0 \\
\hline 149.466702 & 1.660667 & 0.6359 & 4.91 & 2.08 & 0.318805 & 0.445941 & filament & 1830 & 3 & central & 0 \\
\hline 149.434744 & 1.660370 & 0.5339 & 29.45 & 11.74 & 0.810066 & 0.312929 & cluster & 1177 & 30 & satellite & 0 \\
\hline 150.363551 & 1.661725 & 0.9211 & 1.16 & 0.49 & 0.022983 & 0.097828 & filament & -99 & -99 & isolated & 0 \\
\hline 149.633649 & 1.660999 & 0.6832 & 2.21 & 0.91 & 0.001875 & 0.008153 & field & 2237 & 2 & central & 0 \\
\hline 150.332107 & 1.661299 & 1.0417 & 0.76 & 0.35 & 0.010454 & 0.027575 & field & 5763 & 2 & satellite & 0 \\
\hline 149.489826 & 1.660462 & 0.7497 & 1.48 & 0.59 & 0.004759 & 0.020155 & field & -99 & -99 & isolated & 0 \\
\hline 149.420016 & 1.661311 & 0.9977 & 0.59 & 0.26 & 0.012427 & 0.015011 & field & 5302 & 2 & satellite & 0 \\
\hline 150.033681 & 1.661263 & 0.8750 & 3.24 & 1.32 & 0.068370 & 0.040883 & field & 3890 & 2 & satellite & 0 \\
\hline 149.822392 & 1.661513 & 0.9007 & 0.23 & 0.09 & 0.000000 & 0.000670 & field & -99 & -99 & isolated & 0 \\
\hline 150.309442 & 1.661536 & 1.1818 & 1.90 & 1.04 & 0.077422 & 0.058708 & field & -99 & -99 & isolated & 0 \\
\hline 149.807899 & 1.660575 & 0.5954 & 2.26 & 0.98 & 0.177001 & 0.313080 & filament & 1559 & 10 & satellite & 0 \\
\hline 149.458808 & 1.660600 & 0.6356 & 5.98 & 2.53 & 0.395283 & 0.498088 & filament & 1830 & 3 & satellite & 0 \\
\hline 150.161337 & 1.661601 & 0.7401 & 6.60 & 2.63 & 0.187206 & 0.146879 & cluster & 2501 & 23 & satellite & 0 \\
\hline 149.802478 & 1.661148 & 0.6603 & 2.38 & 1.00 & 0.050753 & 0.129584 & filament & 1997 & 3 & satellite & 0 \\
\hline 149.810858 & 1.662660 & 1.1455 & 1.00 & 0.52 & 0.037894 & 0.043417 & filament & -99 & -99 & isolated & 0 \\
\hline 149.421077 & 1.661087 & 0.5080 & 8.61 & 3.43 & 0.464037 & 0.186550 & cluster & 1135 & 5 & satellite & 0 \\
\hline 150.137783 & 1.662048 & 1.1956 & 0.65 & 0.36 & 0.003127 & 0.003832 & field & -99 & -99 & isolated & 0 \\
\hline 149.425513 & 1.662000 & 0.5735 & 2.34 & 0.98 & 0.006330 & 0.137193 & filament & 1420 & 3 & satellite & 0 \\
\hline 150.321295 & 1.662851 & 1.1326 & 2.22 & 1.12 & 0.109606 & 0.076917 & field & 6438 & 2 & satellite & 0 \\
\hline 150.315566 & 1.661740 & 0.5327 & 7.43 & 2.96 & 0.385201 & 0.576236 & filament & 1134 & 52 & satellite & 0 \\
\hline 149.959531 & 1.661993 & 0.3744 & 13.25 & 5.31 & 0.234709 & 0.058717 & cluster & 412 & 47 & satellite & 0 \\
\hline 149.942584 & 1.662862 & 1.0847 & 1.43 & 0.69 & 0.024018 & 0.091628 & filament & -99 & -99 & isolated & 0 \\
\hline 150.264656 & 1.663255 & 0.9347 & 2.48 & 1.06 & 0.026360 & 0.073873 & filament & 4516 & 6 & satellite & 0 \\
\hline 150.078485 & 1.662787 & 0.7150 & 2.52 & 1.02 & 0.005093 & 0.002531 & field & 2417 & 5 & satellite & 0 \\
\hline 149.769251 & 1.663466 & 0.9346 & 1.68 & 0.72 & 0.102289 & 0.076040 & field & 4515 & 2 & satellite & 0 \\
\hline 150.333574 & 1.663059 & 1.1175 & 2.61 & 1.28 & 0.094398 & 0.039504 & field & 6147 & 3 & central & 0 \\
\hline 150.263827 & 1.662800 & 1.1122 & 2.57 & 1.26 & 0.030129 & 0.021809 & field & -99 & -99 & isolated & 0 \\
\hline 149.518530 & 1.662758 & 0.9833 & 1.95 & 0.84 & 0.107281 & 0.082587 & field & -99 & -99 & isolated & 0 \\
\hline 149.831415 & 1.662806 & 0.8337 & 3.69 & 1.47 & 0.021558 & 0.014761 & field & 3219 & 6 & satellite & 0 \\
\hline
\end{tabular}

Note.

${ }^{a}$ Objects that are close to the edge or masked areas are flagged 1. Otherwise, they are flagged 0.

(This table is available in its entirety in machine-readable form.)

each redshift. We clearly see that median $\sigma_{\Delta z /(1+z)} \lesssim 0.01$ out to $z \sim 1.2$, is consistent with the photo- $z$ versus spectroscopic redshift comparison, and small enough for reliable construction of the density field and the cosmic web to $z \sim 1.2$.

\subsection{Stellar Mass, SFR, and Galaxy Classification}

SFRs and stellar masses are based on Laigle et al. (2016), using a SED template fitting procedure similar to that of Ilbert et al. (2015) using UV to mid-IR data. The templates were generated using BC03 (Bruzual \& Charlot 2003), assuming a Chabrier IMF, two metallicities, a combination of exponentially declining and delayed star formation histories, and two extinction curves. Nebular emission line contributions were considered using an empirical relation between the UV and emission line fluxes (Ilbert et al. 2009). The typical stellar mass and SED-based
SFR uncertainties for our sample galaxies to $z \sim 1.2$ are $\Delta M \sim 0.05$ dex and $\Delta \mathrm{SFR}_{\mathrm{SED}} \sim 0.1$ dex, respectively.

To check the reliability of the SED-based SFRs, we compare them with those based on the bolometric IR luminosity for galaxies with a detection in one of Herschel PACS (100 and $160 \mu \mathrm{m})$ and Herschel SPIRE $(250,350$, and $500 \mu \mathrm{m})$ bands (Lee et al. 2013, 2015). This comprises $\sim 10 \%$ of the total galaxies. We find a good agreement between the two SFR indicators, with no significant bias and a median absolute deviation of $\sim 0.25$ dex between them.

The $3 \sigma$ magnitude limit of the survey $\left(K_{s}=24\right.$; Laigle et al. 2016) results in a variable stellar mass completeness limit at different redshifts. Using the empirical method originally developed by Pozzetti et al. (2010; see also Ilbert et al. 2013; Darvish et al. 2015b), we estimate the stellar mass completeness limit by associating a limiting mass to each galaxy at each 


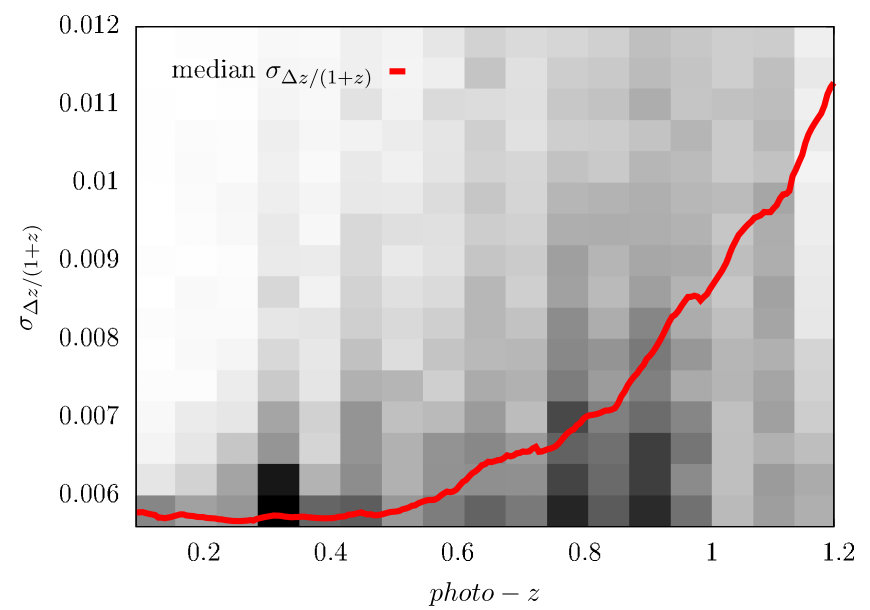

Figure 2. Photo- $z$ uncertainties, $\sigma_{\Delta z /(1+z)}$, as a function of redshift for our sample galaxies. Red line shows the median photo- $z$ uncertainties. We see that the median $\sigma_{\Delta z /(1+z)} \lesssim 0.01$ out to $z \sim 1.2$, small enough for reliable construction of the density field and the cosmic web in this work to $z \sim 1.2$.

redshift. This limiting mass corresponds to the stellar mass that the galaxy would require to have to be detected at its redshift, if its apparent magnitude were equal to the magnitude limit of $K_{s}=24$ :

$$
\log \left(M_{\text {limit }} / M_{\odot}\right)=\log \left(M / M_{\odot}\right)+0.4\left(K_{s}-24\right) .
$$

At each redshift, we define the mass completeness limit as the stellar mass for which $90 \%$ of galaxies have their limiting mass below it. The stellar mass completeness limit also depends on the galaxy type and is higher for quiescent systems. In this study, we rely on the mass completeness limit for quiescent galaxies.

We separate quiescent galaxies from SF systems using restframe NUV $-r^{+}$versus $r^{+}-J$ color-color plot, with quiescent galaxies satisfying the color selection NUV $-r^{+}>3.1$ and NUV $-r^{+}>3\left(r^{+}-J\right)+1$ (Ilbert et al. 2013). Figure 1 shows the estimated mass completeness limit for all the galaxies and the quiescent systems, and our sample of galaxies selected for this study (Section 2).

\subsection{Density Field Construction}

The density field construction is fully described in Darvish et al. (2015b). Here, we provide a summary and some revisions to the original method. We estimate the density field for a series of overlapping redshift slices ( $z$-slice) with variable widths to $z=1.2$. As suggested by Malavasi et al. (2016), $z$-slice widths are selected to be within $\pm 1.5 \sigma_{\Delta z /(1+z)}$ from the center of each redshift (this is slightly different than the widths originally defined in Darvish et al. (2015b)). Then, for each $z$-slice, we associate a weight to each galaxy by measuring the percentage of the photo- $z$ probability distribution function (PDF) of the galaxy that lies within the boundaries of each $z$-slice. This shows the likelihood of a galaxy belonging to that $z$-slice. At each $z$-slice, all galaxies that have weights $\geqslant 10 \%$ are selected for density estimation. The incorporation of the weights tends to significantly diminish the projection effect due to the uncertainties in the photo-zs.

Through extensive simulations, Darvish et al. (2015b) showed that adaptive kernel smoothing (also see Scoville et al. 2007a, 2013) and Voronoi tessellation perform better in constructing the density field compared to other estimators such as the nearest neighbor and Delaunay triangulation. Here, we use the weighted adaptive kernel smoothing (where weights are

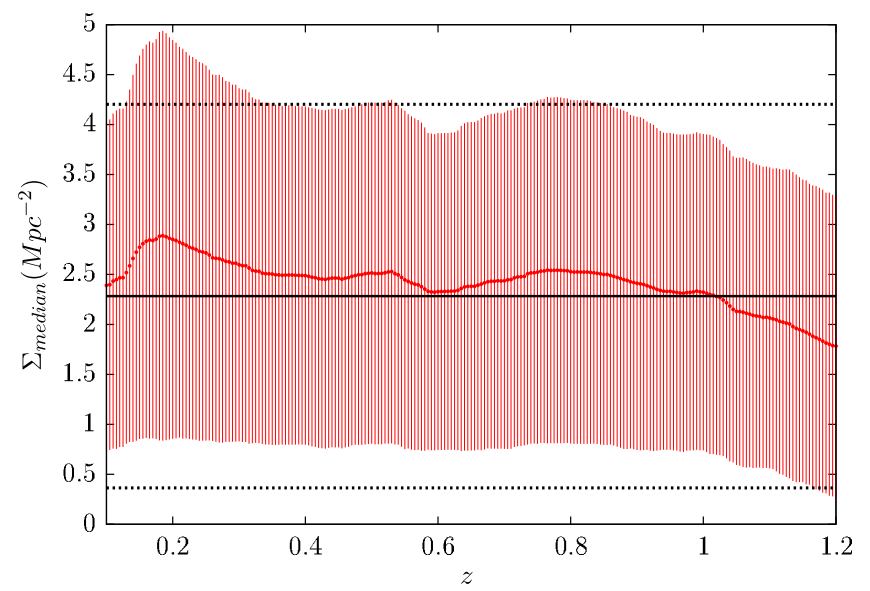

Figure 3. Red symbols show the estimated median density as a function of redshift for our sample galaxies. To minimize the cosmic variance, median densities are estimated within \pm 0.2 redshift intervals at each redshift. The uncertainties are $1.4826 \times$ the median absolute deviation from the median values. The black solid line shows the estimated median density over the whole field $(0.1<z<1.2)$ and the black dashed lines show its upper and lower uncertainties. We find that within the uncertainties, the median values do not change much with redshift. This emphasizes a volume-limited-like sample selection to avoid any unrealistic underestimation of the density values at higher $z$ as a result of missing fainter, less-massive galaxies.

the assigned galaxy weights explained before) using a 2D Gaussian kernel whose width adaptively changes over the field according to the local density of galaxies. The global smoothing width is selected to be $0.5 \mathrm{Mpc}$, which corresponds to the typical virial radius for X-ray groups and clusters in the COSMOS field (Finoguenov et al. 2007; George et al. 2011).

In constructing the density field, we use our sample of galaxies, which is similar to a volume-limited sample. This avoids any unrealistic underestimation of the density estimates at higher redshift as less-massive fainter galaxies would be missed at those redshifts. Figure 3 shows the median density as a function of redshift, along with the median density estimated from the whole field $\left(2.28 \mathrm{Mpc}^{-2}\right)$. We see that within the uncertainties, the median values do not change much with redshift. To minimize the cosmic variance, median densities are estimated within \pm 0.2 redshift intervals at each redshift. The uncertainties are $1.4826 \times$ the median absolute deviation from the median values. Within the uncertainties, our estimated median values are also similar to the density cut used by Erfanianfar et al. (2016) to separate field and group galaxies $\left(\Sigma_{\text {cut }}=3 \mathrm{Mpc}^{-2}\right)$, even though they used different selection functions than us.

Figures 4(a), 5(a), and 6(a) show the galaxies selected for density estimation for $z$-slices centered at $z=0.360,0.530$, and 0.980 , respectively. The size of each point is scaled with the weight of that galaxy. The estimated density field for these slices are shown in Figures 4(b), 5(b), and 6(b). We finally interpolate our sample galaxies to the estimated density field using their angular position and photo- $z$ PDF. The $z$-slice for each galaxy is the one at which its weight maximizes. Table 1 lists all of our sample galaxies with their estimated density values. The overdensity defined as the density with respect to the median density at each redshift $\left(\Sigma / \Sigma_{\text {median }}\right)$ is also given.

\subsection{Cosmic Web Extraction}

\subsubsection{The Method}

We extract the components of the cosmic web (filaments and clusters) in the density field using the $2 \mathrm{D}$ version of the 


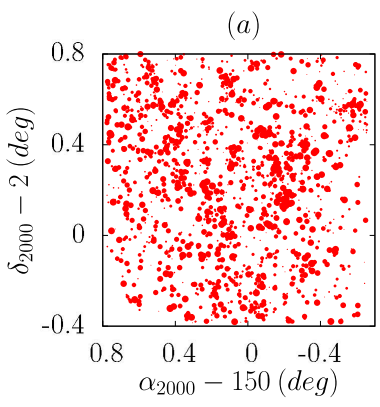

(c)

(e)
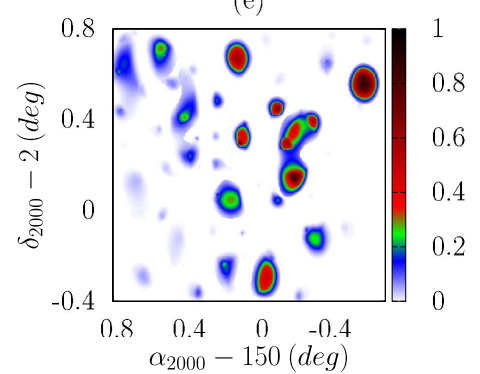

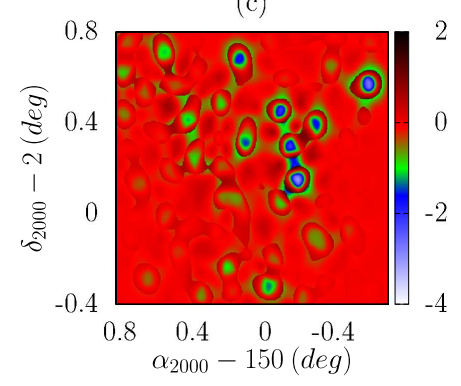

(b)

(d)

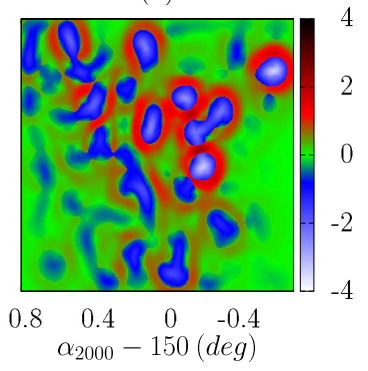

(f)

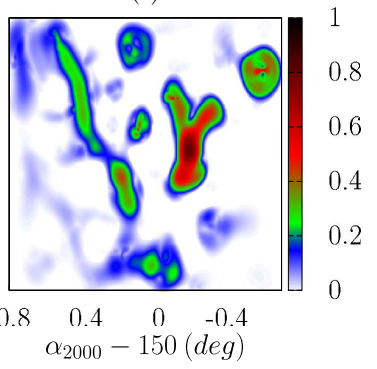

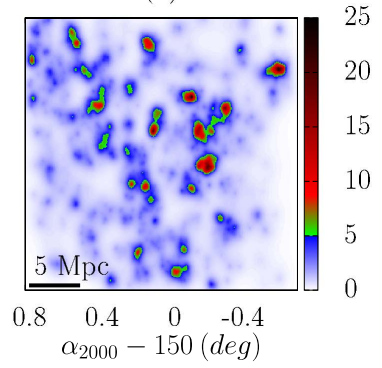

Figure 4. (a) Galaxies selected for density estimation for a $z$-slice centered at $z=0.360$. The size of each point is proportional to the weight assigned to each galaxy (Section 3.3). (b) Density field estimated using the weighted adaptive kernel smoothing estimator (Section 3.3) for the selected galaxies. (c) $\lambda_{1}$ eigenvalue map of the Hessian matrix evaluated at the physical scale $L=1.00 \mathrm{Mpc}$. (d) $\lambda_{2}$ eigenvalue map of the Hessian matrix evaluated at the physical scale $L=1.00 \mathrm{Mpc}$, assuming $\left|\lambda_{2}\right| \geqslant\left|\lambda_{1}\right|$. (e) Final cluster signal map for the $z$-slice at $z=0.360$ after taking the multi-scale nature of the cosmic web into account. Note that for an ideal cluster we have $\left|\lambda_{1}\right| \approx\left|\lambda_{2}\right|$. (f) Final filament signal map for the $z$-slice at $z=0.360$ after taking the multi-scale nature of the cosmic web into account. Note that for an ideal filament we have $\left|\lambda_{1}\right| \ll\left|\lambda_{2}\right|$.

Multi-scale Morphology Filter algorithm (Aragón-Calvo et al. 2007a; Darvish et al. 2014). In this method, we associate a filament and a cluster signal to each point (values between 0 and 1) in the density field based on the resemblance of the local geometry of that point to a filament or a cluster. The local geometry of each point is calculated based on the signs and ratio of eigenvalues of the Hessian matrix $H(\boldsymbol{r})$ which is the second-order derivative of the density field $\Sigma(\boldsymbol{r})$ :

$$
H(\boldsymbol{r})=\left[\begin{array}{ll}
\nabla_{x x} \Sigma(\boldsymbol{r}) & \nabla_{x y} \Sigma(\boldsymbol{r}) \\
\nabla_{y x} \Sigma(\boldsymbol{r}) & \nabla_{y y} \Sigma(\boldsymbol{r})
\end{array}\right],
$$

where $\nabla_{i j}$ s denote the second-order derivatives in the $i$ and $j$ directions.

Since structures in the density field (filaments and clusters) have different physical sizes, we build a scale-independent structure map by smoothing the surface density field over a range of physical scales and eventually selecting the greatest cluster and filament signal among all the various signal values at different physical scales. In practice, we use a 2D Gaussian

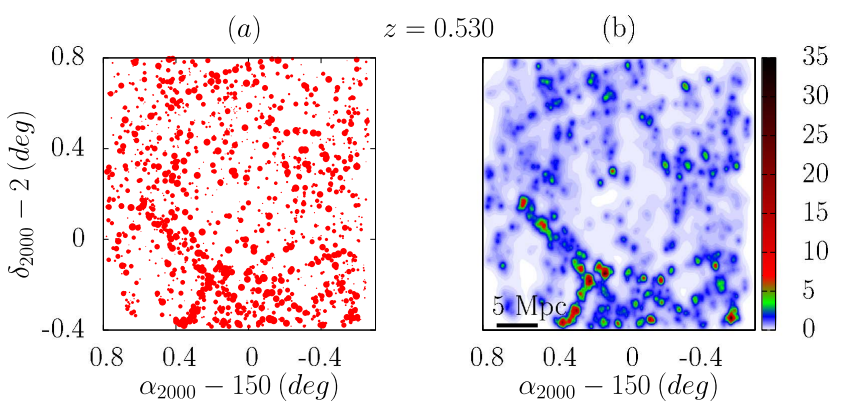

(c)

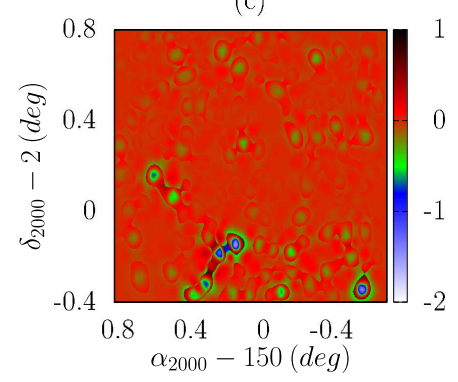

(d)

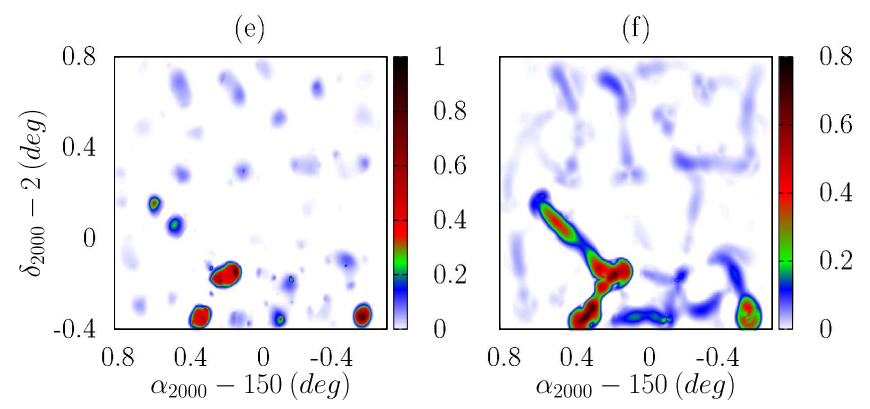

Figure 5. Similar to Figure 4 but for a $z$-slice centered at $z=0.530$.

smoothing function with physical scales $L=0.25,0.50,0.75$, $1.00,1.50$, and $2.00 \mathrm{Mpc}$. Therefore, the components of the Hessian matrix at scale $L$ are

$$
\begin{aligned}
H_{L}(\boldsymbol{r}) & =\Sigma \otimes \nabla_{i j} G_{L} \\
& =\int d \boldsymbol{r}^{\prime} \Sigma\left(\boldsymbol{r}^{\prime}\right) \frac{\left(x_{i}-x_{i}^{\prime}\right)\left(x_{j}-x_{j}^{\prime}\right)-\delta_{i j} L^{2}}{L^{4}} G_{L}\left(\boldsymbol{r}^{\prime}, \boldsymbol{r}\right),
\end{aligned}
$$

where $x_{1}, x_{2}=x, y, x_{1}^{\prime}, x_{2}^{\prime}=x^{\prime}, y^{\prime}, \delta_{i j}$ is the Kronecker delta and $G_{L}\left(\boldsymbol{r}^{\prime}, \boldsymbol{r}\right)$ is our 2D Gaussian smoothing function at scale $L$ :

$$
G_{L}\left(\boldsymbol{r}^{\prime}, \boldsymbol{r}\right)=\frac{1}{2 \pi L^{2}} \exp \left(-\frac{\left|\boldsymbol{r}^{\prime}-\boldsymbol{r}\right|}{2 L^{2}}\right) .
$$

The eigenvalues of the Hessian matrix at each point are a measure of the rate of change of the density field gradient in their corresponding eigenvector direction. Therefore, if the local geometry of a point resembles a cluster, one expects the local eigenvalues to be negative and their ratio close to one. For a filament, one expects the eigenvalue corresponding to the eigenvector perpendicular to the filament to be negative and the ratio of the smaller eigenvalue (in the direction of the filament where the rate of change of density values is small) to the larger one to be close to zero. Figures 4(c) and (d), 5(c) and (d), and 6(c) and (d) show the eigenvalue maps $\left(\lambda_{1}\right.$ and $\left.\lambda_{2}\right)$ at the 
physical scale $L=1.00 \mathrm{Mpc}$ for $z$-slices at $z=0.360,0.630$, and 0.980 , respectively. Given these, if $\lambda_{1}$ and $\lambda_{2}$ are the eigenvalues and $\left|\lambda_{2}\right| \geqslant\left|\lambda_{1}\right|$, we define the morphology mask $\varepsilon$ for clusters and filaments at each point in the density field as

$$
\begin{array}{rll}
\varepsilon_{\text {cluster }}=0 & \text { if } \quad \lambda_{1}>0 \quad \text { or } \quad \lambda_{2}>0 ; 1 \text { otherwise } \\
\varepsilon_{\text {filament }}=0 & \text { if } & \lambda_{2}>0 ; 1 \quad \text { otherwise. }
\end{array}
$$

For those points in the density field that pass the above conditions $(\varepsilon=1)$, we quantify the degree of resemblance to a cluster or a filament by defining the function

$$
\begin{aligned}
D_{\text {cluster }} & =\frac{\left|\lambda_{1}\right|}{\left|\lambda_{2}\right|} \\
D_{\text {filament }} & =1-\frac{\left|\lambda_{1}\right|}{\left|\lambda_{2}\right|} .
\end{aligned}
$$

Note that for an ideal cluster $\left(\left|\lambda_{1}\right| \approx\left|\lambda_{2}\right|\right)$, we have $D_{\text {cluster }} \approx$ 1 and $D_{\text {filament }} \approx 0$, whereas for an ideal filament $\left(\left|\lambda_{1}\right| \ll\left|\lambda_{2}\right|\right)$, we get $D_{\text {cluster }} \approx 0$ and $D_{\text {filament }} \approx 1$.

Using the already-defined function $D$, we define the following function $M$ for clusters and filaments (Frangi et al. 1998):

$$
\begin{aligned}
M_{\text {cluster }} & =\exp \left(-\frac{D_{\text {filament }}}{2 \beta^{2}}\right) \\
M_{\text {filament }} & =\exp \left(-\frac{D_{\text {cluster }}}{2 \beta^{2}}\right),
\end{aligned}
$$

where $\beta$ controls the aggressiveness of feature selection. Here we choose $\beta=0.5$ as a typical value (Frangi et al. 1998; Aragón-Calvo et al. 2007a). Note again that for clusters, $D_{\text {filament }}$ is small and therefore $M_{\text {cluster }}$ is large, whereas for filaments, $D_{\text {cluster }}$ is small and hence $M_{\text {filament }}$ is large.

Another important piece of information that we can use to enhance the detection of structures is that features of our interest (clusters and filaments) are more pronounced in the density field than the overall background distribution. If we do not take this into account, random background fluctuations may result in unrealistic features. For the background, the magnitude of second-order derivatives (and hence eigenvalues) is small due to the lack of contrast. Therefore, we use the norm of the Hessian matrix by defining the function

$$
I=1-\exp \left(-\frac{\mathrm{Norm}^{2}}{2 c^{2}}\right),
$$

where Norm $=\sqrt{\lambda_{1}^{2}+\lambda_{2}^{2}}$ and $c$ controls the sensitivity of this function. Here we use $c=0.5 \times$ maximum (Norm) at each $z$ slice (Frangi et al. 1998).

Finally, at each scale $L$, the signal map is defined as

$$
S_{L}=\varepsilon \otimes M \otimes I
$$

and eventually every pixel of the final signal map $S$ gets the maximum of all the corresponding pixels at different physical scales. That is,

$$
S=\max \left(S_{L}\right) .
$$

Figures 4(e) and (f), 5(e) and (f), and 6(e) and (f) show the final cluster $\left(S_{\text {cluster }}\right)$ and filament $\left(S_{\text {filament }}\right)$ signal maps for the $z$ slices at $z=0.360,0.630$, and 0.980 , respectively. Filament and cluster signal values for our sample galaxies are given in Table 1.
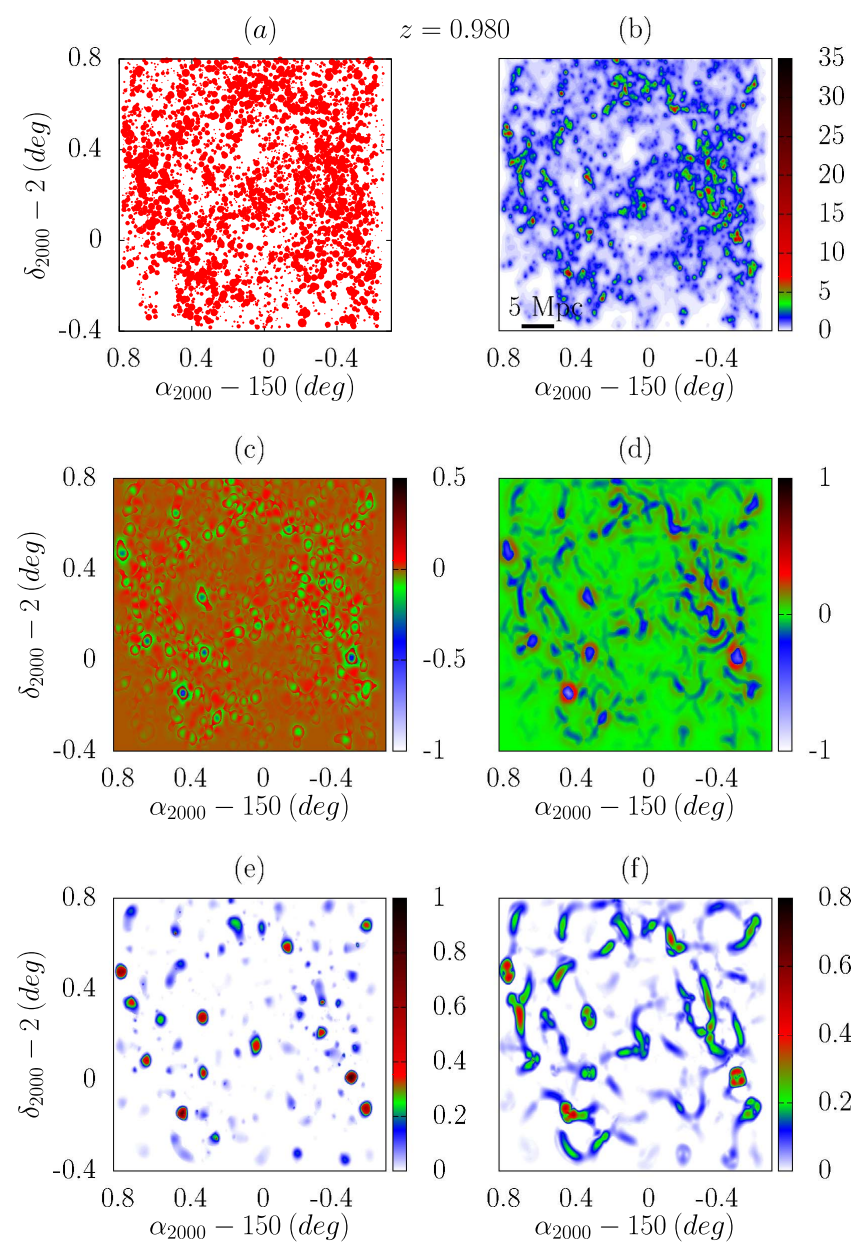

Figure 6. Similar to Figure 4 but for a $z$-slice centered at $z=0.980$.

\subsubsection{Filament, Cluster, and Field Selection}

For each $z$-slice, there is a filament and a cluster signal assigned to each point which is a value between 0 and 1 . Choosing a very high signal value results in a small sample size and disregards possible real structures with small signal values, whereas a very small signal is prone to contamination from unreal features and noise in the density field. Therefore, an appropriate trade-off signal cut for filaments and clusters should be chosen.

Following Aragón-Calvo et al. (2007a), we select the appropriate cluster and signal cuts at different redshifts. For clusters, if we plot the fraction of volume occupied by below the cluster signal $\left(V_{<S_{c}}\right)$ as a function of the cluster signal $\left(S_{c}\right)$, we see a monotonically increasing function that can be described by a two power-law behavior (Figure 7(a) for the $z$-slice at $z=0.630$ ). The cluster signal that corresponds to the intersection of the two power-law functions is selected as the best cluster signal cut. Figure 7(a) shows this for one of the $z$ slices. The best cluster signal cut varies from slice to slice and is likely affected by the cosmic variance. Hence, we fit a linear function to the best signal cut at different redshifts and use the fitted line $(0.0639 z+0.1142)$ for selection (Figure $7(b))$. The typical best cluster signal cut is in the range $\sim 0.1-0.2$.

For filaments, the number of individual filaments $\left(N_{f}\right)$ at very small filament signal cut $\left(S_{f}\right)$ is small because pixels tend to percolate and form large filaments. At very large $S_{f}$ values, $N_{f}$ is 
also small because only a small fraction of pixels pass the selection cut. Therefore, if we plot $N_{f}$ versus $S_{f}$, it maximizes at some $S_{f}$ value (Figure 7(c) for the $z$-slice at $z=0.630$ ). We use this $S_{f}$ corresponding to the peak of $N_{f}$ as the best filament signal cut. Similar to the cluster selection, we use the fitted line $(0.0253 z+0.0035)$ results to select the best filament signal cut at different redshifts (Figure 7(d)). The typical best filament signal cut is in the range $\sim 0.01-0.04$.

At each redshift, all of the galaxies that have their cluster signal greater than (or equal to) the best cluster signal cut at that redshift and their cluster signal greater than (or equal to) their filament signal are selected as cluster galaxies. We use the remaining points to impose the filament selection. Among the remaining points, all of the galaxies that have their filament signal greater than (or equal to) the best filament signal cut at that redshift and their filament signal greater than (or equal to) their cluster signal are selected as filament galaxies. Eventually, the final remaining points that do not satisfy both filament and cluster selections are chosen as the field. Table 1 contains the cosmic web environment of our sample galaxies.

\subsubsection{Caveats and Limitations}

We note that in 2D it is impossible to distinguish between filaments and walls using the MMF algorithm. Therefore, some filament galaxies could potentially be galaxies located in walls. We also mention that due to the 2D nature of our approach, filaments oriented radially in the line of sight might be misclassified as clusters. As we discussed in Section 3.4.1, the smallest scale that is probed here is $L=0.25 \mathrm{Mpc}$. Given the mass cut used in this work, it is likely that some very thin filaments containing less-massive fainter galaxies are not detected (e.g., see Alpaslan et al. 2014b), potentially leading to an increase in the field population.

\subsection{Central, Satellite, and Isolated Classification}

We select a sample of galaxy groups and use it to observationally classify central and satellite galaxies in our data set. We select the most massive galaxy in each group as a central and the rest as satellites. Galaxies that are not associated with any galaxy group (isolated) are either centrals whose satellites, in principle, are too faint to be detected in our volume-limited sample or they are ejected satellites orbiting beyond their halo's virial radius (see, e.g., Wetzel et al. 2014). We rely on our sample galaxies (our volume-limited-like sample) to identify groups. We use the commonly used friendsof-friends algorithm (Huchra \& Geller 1982; also see Duarte \& Mamon 2014 and the references therein), by linking galaxies whose redshift difference and angular separations are less than some critical values. Two galaxies $i$ and $j$ with redshifts $z_{i}$ and $z_{j}$ and angular separation $\Delta \theta_{i j}$ are linked to each other if they satisfy the following conditions:

$$
\begin{gathered}
D_{c}(z) \Delta \theta_{i j} \leqslant b_{\perp} n(z)^{-1 / 2} \\
\left|z_{i}-z_{j}\right| \leqslant \alpha \sigma_{\Delta z /(1+z)},
\end{gathered}
$$

where $D_{c}(z)$ is the comoving distance at $z$ (average redshift of galaxies $i$ and $j), n(z)$ is the median number density of galaxies at $z$ (Figure 3 ), $b_{\perp}$ is the projected linking length in units of the median projected intergalaxy separation at $z$, and $\alpha$ is a parameter that controls the line-of-sight linking of galaxies as a function of the typical photo- $z$ uncertainties at $z$.
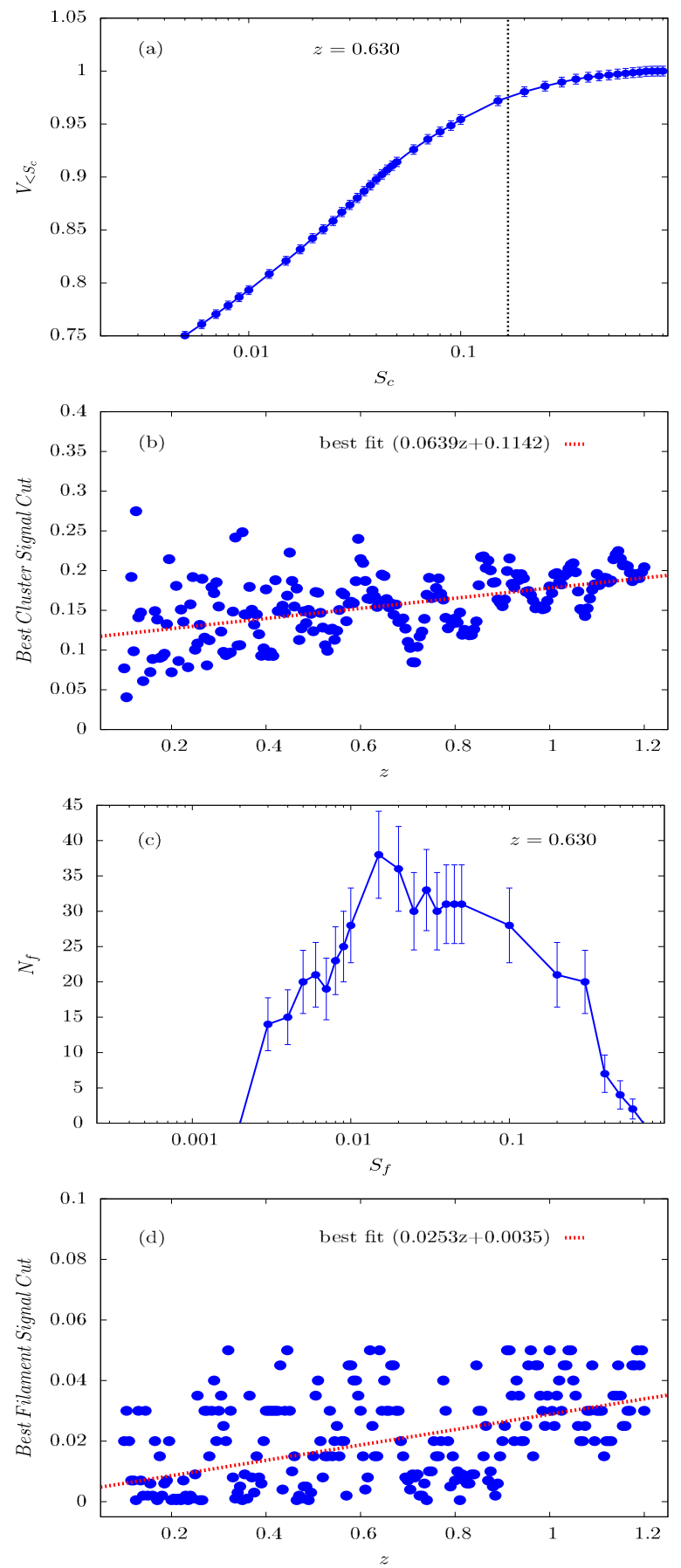

Figure 7. (a) Fraction of volume occupied by below the cluster signal $\left(V_{<S_{c}}\right)$ as a function of the cluster signal $\left(S_{c}\right)$ for the $z$-slice at $z=0.630$. We see a monotonically increasing function that can be described by a two power-law behavior. The cluster signal that corresponds to the intersection of the two power-law functions (shown as the black dashed line) is selected as the best cluster signal cut. (b) Best cluster signal cut as a function of redshift. It varies from slice to slice and is affected by the cosmic variance. We fit a linear function to the best signal cut at different redshifts and use the fitted line, shown with a red dashed line, for selection of cluster galaxies. (c) Number of individual filaments $\left(N_{f}\right)$ as a function of filament signal cut $\left(S_{f}\right)$ for the $z$-slice at $z=0.630$. We use the $S_{f}$ corresponding to the peak of $N_{f}$ as the best filament signal cut (Section 3.4.2). (d) Best filament signal cut as a function of redshift. We fit a linear function to the best signal cut at different redshifts and use the fitted line, shown with a red dashed line, for selection of filament galaxies.

The appropriate values for $b_{\perp}$ and $\alpha$ are key in selection of our galaxy groups. Small linking lengths tend to break groups into many subcomponents, whereas large linking lengths tend 


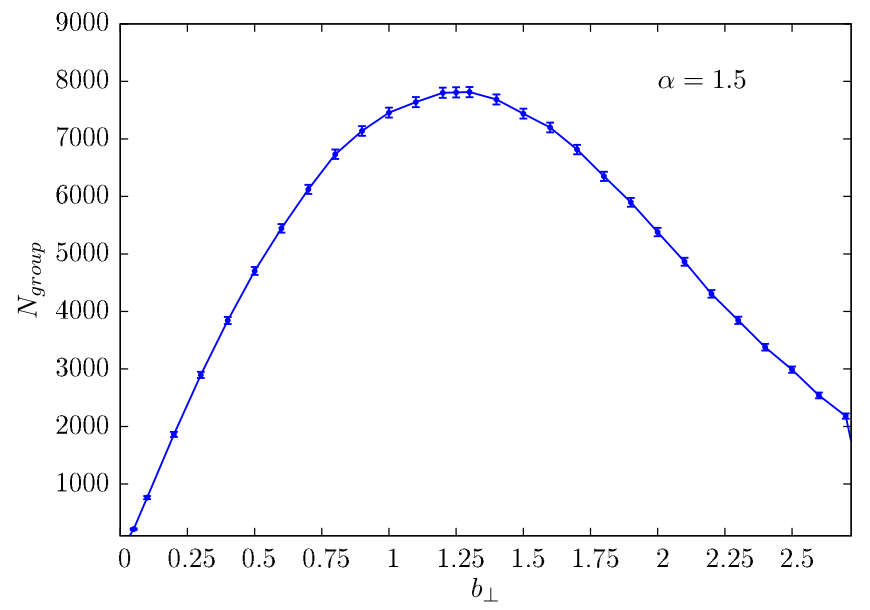

Figure 8. Number of groups $N_{\text {group }}$ as a function of the projected linking length in units of the median projected intergalaxy separation $\left(b_{\perp}\right)$ for a fixed $\alpha=1.5$ (Section 3.5). The number of groups for small $b_{\perp}$ values is low because only a small number of galaxies are too close to link together. On the other hand, for large values of $b_{\perp}, N_{\text {group }}$ is also small because many galaxies link together to form percolated structures. By fixing $\alpha=1.5$, the number of selected groups maximizes at $b_{\perp} \sim 1.3$ and we use it as a suitable trade-off linking length for our group selection.

to percolate and link different groups into a single larger one. Therefore, depending on the science of interest, trade-off linking lengths will be selected.

Here, we use $b_{\perp}=1.3$ and $\alpha=1.5$. The selection of latter is based on our discussion in Section 3.1 and Malavasi et al. (2016). Similar to our discussion in Section 3.4.2, the number of groups $\left(N_{\text {group }}\right)$ for small $b_{\perp}$ values is low because only a small number of galaxies are too close to link together. On the other hand, for large values of $b_{\perp}, N_{\text {group }}$ is also small because many galaxies link together to form percolated structures. By fixing $\alpha=1.5$, the number of selected groups maximizes at $b_{\perp}$ $\sim 1.3$ and we use it as a suitable, trade-off linking length (see Figure 8). We note that fine-tuning the above-mentioned parameters does not significantly change our results. Table 1 lists the group ID, number of group members, and the galaxy type (satellite, central, isolated) of our sample.

\section{Results and Discussion}

\subsection{Basic Properties of the Cosmic Web}

The median density of our sample galaxies in clusters, filaments, and the field is $8.61,3.09$, and $1.53 \mathrm{Mpc}^{-2}$, respectively. Therefore, the median density value for the whole field estimated in Section $3.3\left(2.28 \mathrm{Mpc}^{-2}\right)$ corresponds mostly to filament- and field-like environments. However, there is substantial overlap between densities of galaxies in different environments, meaning that a pure density-based definition of cosmic web environment is not fully adequate to identify different structures (Aragón-Calvo et al. 2010b; Darvish et al. 2014).

Our cluster, filament, and field environments occupy $1.6 \%$, $21.6 \%$, and $76.8 \%$ of the total volume, respectively. These are in good agreement with $z=0$ simulations of Cautun et al. $(2014 ;<0.1 \%, 24 \%$, and $77 \%$ for clusters, filaments + walls, and voids, respectively) and Aragón-Calvo et al. (2010b; 0.4\%, $13.7 \%$, and $85.9 \%$ for clusters, filaments +walls, and the field, respectively). However, the cluster volume fraction is higher than these simulations likely because our cluster sample contains some line-of-sight filaments misclassified as clusters,
Table 2

Basic Properties of the Cosmic Web

\begin{tabular}{lccr}
\hline \hline & Cluster & Filament & Field \\
\hline Median density $\left(\mathrm{Mpc}^{-2}\right)$ & 8.61 & 3.09 & 1.53 \\
Volume fraction $(\%)$ & 1.6 & 21.6 & 76.8 \\
Galaxy number fraction $(\%)$ & 10.9 & 40.8 & 48.3 \\
Galaxy stellar mass fraction $(\%)$ & 14.3 & 41.1 & 44.6 \\
\hline
\end{tabular}

as discussed in Section 3.4.3. Another possibility might be due to the cosmic variance. For example, the Cautun et al. (2014) simulation volume is $\sim 40$ times larger than that of the COSMOS field to $z=1.2$. It is also worth noting that depending on the selection function and the methods used, these statistics vary. For example, cluster (void) volume fraction in the literature can be as low as $<0.1 \%(<10 \%)$ or as high as $\sim 10 \%(>90 \%$; see Cautun et al. 2014 for references).

The fraction of our sample galaxies in different environments is $10.9 \%, 40.8 \%$, and $48.3 \%$ in clusters, filaments, and the field, respectively. In terms of the stellar mass, the stellar mass fraction of our sample galaxies $\left(\log \left(M / M_{\odot}\right) \geqslant 9.6\right)$ is $14.3 \%, 41.1 \%$, and $44.6 \%$ in cluster, filament, and field environments, respectively. We clearly see that although clusters occupy only a small fraction of the volume, they contain a measurable number and stellar mass fraction of galaxies used in this study. We also find that the overall fraction of our $\log \left(M / M_{\odot}\right) \geqslant 9.6$ galaxies populating clusters increases from $9.5 \pm 0.2 \%$ at $0.8 \leqslant z \leqslant 1.2$ to $16.2 \pm 0.5 \%$ at $0.1 \leqslant z \leqslant 0.5$, consistent with simulations. Table 2 lists basic properties of the cosmic web.

\subsection{SF Activity in the Cosmic Web}

Figure 9(a) shows the median SFR for galaxies in different parts of the cosmic web from the field to clusters for different redshift bins. Error bars are estimated using the bootstrap resampling, added in quadrature to typical observational uncertainties and uncertainties due to the cosmic variance. We clearly see a gradual decline in the median SFR from the field to clusters at $z \lesssim 0.8$ but at higher redshifts $(z \gtrsim 0.8)$, the trend flattens out. The decline in the median SFR from the field to filaments is not significantly large but the SFR difference between cluster galaxies and those located in other regions of the cosmic web is quite evident at $z \lesssim 0.8$.

We further investigate this relation for satellite, central, and isolated galaxies as shown in Figures 9(b)-(d). For satellites, the trends are very similar to the overall population of galaxies, indicating the dominance of satellites in determining the general trends. For centrals and at $z \lesssim 0.8$, we also see a decline in the median SFR from the field to clusters although the decline is not as sharp as that of satellites in the same redshift range. For examples, for satellites at $0.1 \leqslant z \leqslant 0.5$, the median SFR decreases by $\sim 1$ dex as one goes from the field to clusters, whereas centrals show a $\sim 0.5$ dex decline. This shows that the environmental quenching is mostly due to satellites. Isolated galaxies at $z \lesssim 0.8$ show similar trends to centrals. However, at $z \gtrsim 0.8$, their trend resembles that of satellites. Note that the difference between the median SFR of filament and field galaxies is not significant within the uncertainties.

Interestingly, for central galaxies, we still see an environmental (cosmic web) dependence in the median SFR even at 

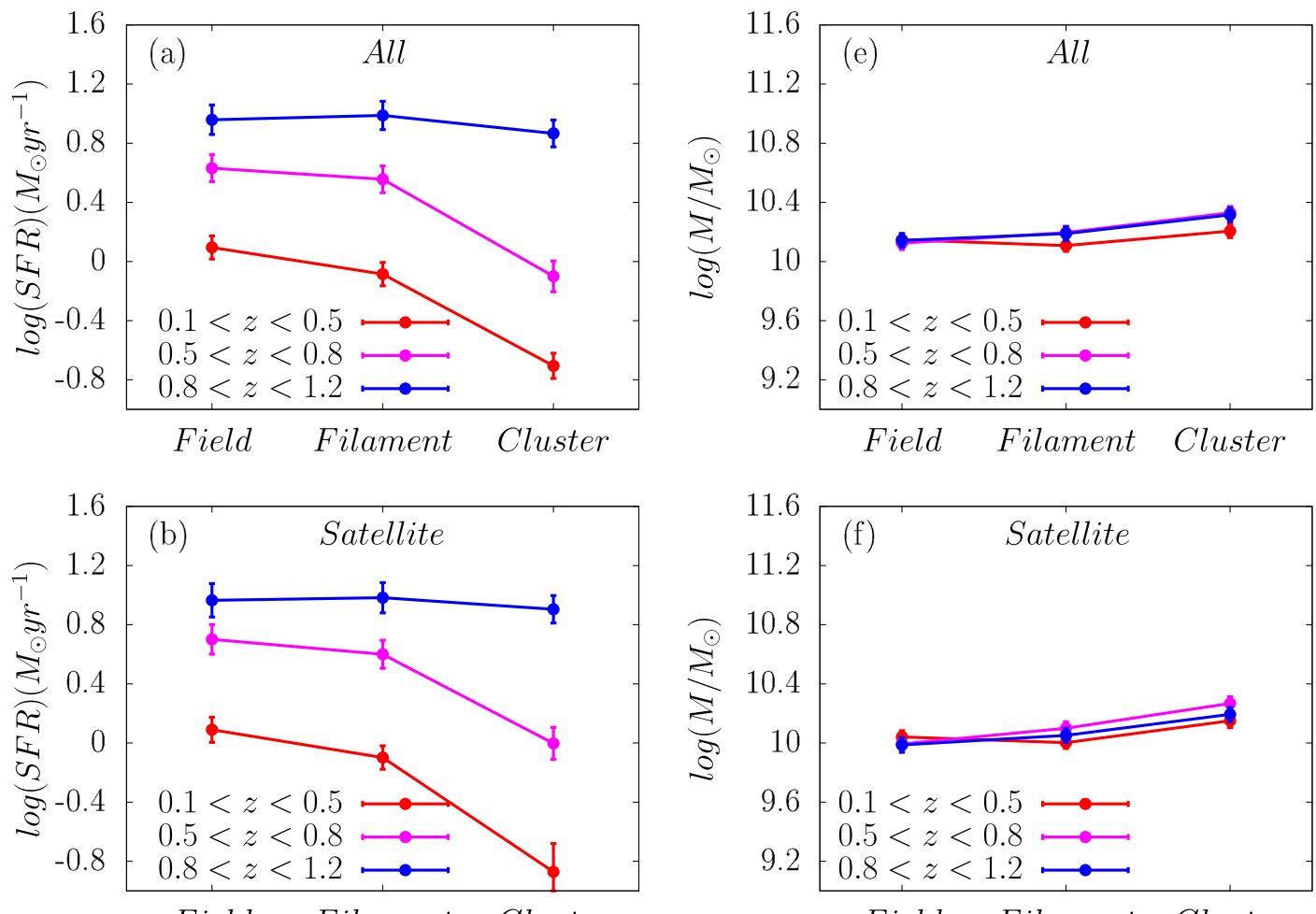

Field Filament Cluster
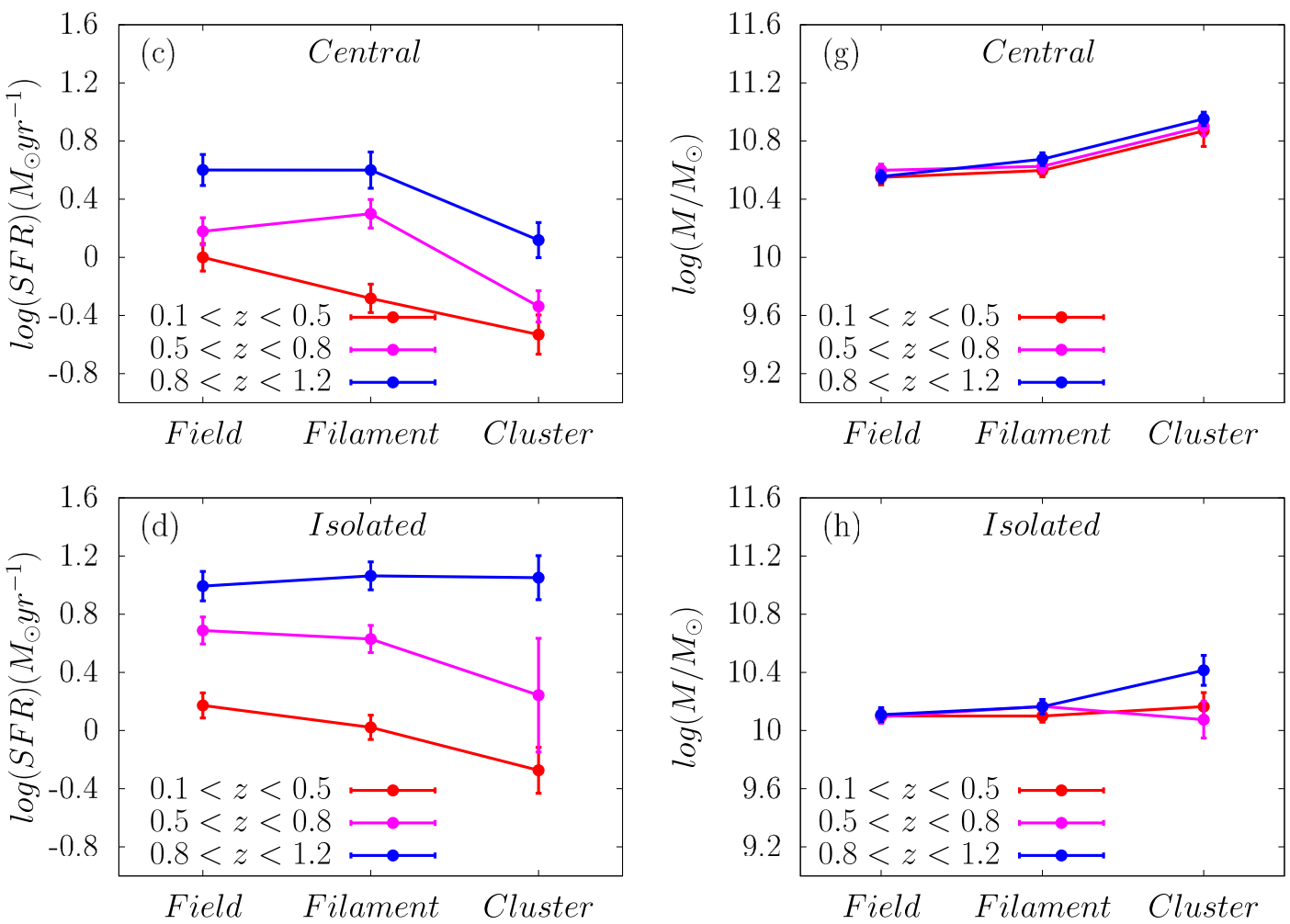

Figure 9. (a) Median SFR for galaxies in different parts of the cosmic web from the field to clusters for different redshift bins. We clearly see a gradual decline in the median SFR from the field to clusters at $z \lesssim 0.8$ but at higher redshifts $(z \gtrsim 0.8)$, the trend flattens out. The decline in the median SFR from the field to filaments is not significantly large but the SFR difference between cluster galaxies and those located in other regions of the cosmic web is quite evident at $z \lesssim 0.8$. (b) Median SFR for satellite galaxies located in different regions of the comic web at different redshifts. These trends are very similar to the overall population of galaxies, indicating the dominance of satellites in determining the general trends. At $0.1 \leqslant z \leqslant 0.5$, the median SFR of satellites decreases by $\sim 1$ dex as one goes from the field to clusters. (c) Median SFR of central galaxies in the comic web at different redshifts. In the whole redshift range considered here, the median SFR of central galaxies decreases by $\sim 0.5$ dex from the field to clusters. (d) Median SFR of isolated galaxies in the comic web at different redshifts. Isolated systems at $z \lesssim 0.8$ show similar trends to centrals, whereas at $z \gtrsim 0.8$, their trend resembles that of satellites. (e)-(h) Median stellar mass for all, satellite, central, and isolated galaxies in the cosmic web, respectively. Within the uncertainties, we see almost no change or a slight increase in some cases $(\sim 0.2-0.3$ dex in maximum) in the median stellar mass of galaxies from the field to clusters. Therefore, stellar mass differences in different parts of the cosmic web cannot much explain these trends or make them even stronger. 
higher redshifts $(z \gtrsim 0.8)$, with a decline of $\sim 0.5 \mathrm{dex}$ in the median SFR from the field to clusters. Satellites and isolated systems do not show any cosmic web dependence at these redshifts.

It is important to consider the role of stellar mass on these trends as well. For example, the decline in the median SFRs from the field to clusters might be simply due to a different stellar mass distribution in different regions of the cosmic web. We investigate this by estimating the median stellar mass in the cosmic web as shown in Figures 9(e)-(h). Within the uncertainties, we see almost no change or a slight increase in most cases ( $\sim 0.2-0.3$ dex in maximum) in the median stellar mass of galaxies from the field to clusters. Therefore, if we control for stellar mass in different parts of the comic web, these trends do not change or become even stronger.

For example, for satellite galaxies at $0.8 \leqslant z \leqslant 1.2$, the median stellar mass increases by $\sim 0.2$ dex from the field to clusters (but with large error bars). If we assume that this increase is dominated by SF (or quiescent) galaxies, the $\sim 0.2$ dex stellar mass enhancement from the field to clusters is equivalent to $\sim 0.2$ dex increase in the SFR, assuming a slope of $\sim 1$ for the main-sequence of SF (or quiescent) galaxies. This means that the median SFR in clusters should in principle decrease by $\sim 0.2$ dex compared to the field when we control for the stellar mass. This suggests that there might still exist a cosmic web dependence of the median SFR for satellite galaxies even at $0.8 \leqslant z \leqslant 1.2$ although the trend is not as strong as those at lower redshifts and the large uncertainties do not allow for a strong conclusion.

The overall decline in the median SFR from the field to clusters can be due to an overall decline in the median SFR of individual galaxies as they fall from the field into clusters through filaments, a decline in the fraction of SF galaxies from the field to clusters or both. We investigate this by focusing on $\mathrm{SF}$ galaxies only. The SF and quiescent separation is based on the color-color plots explained in Section 3.2.

Figures 10(a)-(d) show the median SFR for all SF, satellite $\mathrm{SF}$, central SF, and isolated SF galaxies in the cosmic web. We clearly see that at $z \gtrsim 0.5$, and within the uncertainties, the median SFR for SF centrals, satellites, isolated, and all, do not depend much on the cosmic web. However, at $z \lesssim 0.5$, all satellite, central, and isolated (and all) SF galaxies show a $\sim 0.3-0.4$ dex decline in their median SFR.

Therefore, at $z \gtrsim 0.5$, the decline in the median SFR from the field to clusters for satellites, centrals, and isolated galaxies is mainly due to a change in the fraction of SF and quiescent galaxies, whereas at $z \lesssim 0.5$, the decline is due to a combination of the overall decline in the SFR of individual galaxies and a decline in the fraction of SF galaxies from the field to clusters. However, at $z \lesssim 0.5$ and for central and isolated galaxies, the overall decline in the SFR of individual central and isolated galaxies is the main cause $(\sim 0.4-0.5 \mathrm{dex}$ out of $\sim 0.5$ dex decline can be explained by it), whereas, for satellites, the change in the fraction of $\mathrm{SF} /$ quiescent satellite galaxies is the main driver of this trend $(\sim 0.7 \mathrm{dex}$ out of $\sim 1$ dex decline can be explained by it).

Figure 11 shows this conclusion more clearly. The SF fraction is plotted for all the galaxies, satellites, centrals, and isolated systems in the cosmic web. We clearly see that the SF fraction for the global trend and satellite systems declines from the field to clusters at all the redshifts considered in this study, without a significant evolution. However, for centrals, the SF fraction declines from the field to clusters only at $z \gtrsim 0.5$ and is almost unchanged at $z \lesssim 0.5$ within the uncertainties. Note that within the uncertainties, the change in the SF fraction between field and filament galaxies is not significant.

Given these, most satellite galaxies experience a rapid quenching mechanism as they fall from the field into clusters through the channel of filaments, whereas central and isolated galaxies undergo a slow environmental quenching process at $z \lesssim 0.5$ and a fast mechanism at higher redshifts $(z \gtrsim 0.5)$. We note again that controlling the stellar mass does not much affect (or tends to decease) the median SFRs in clusters compared to the field and filaments. Similar results are also found for the relation between the cosmic web and the sSFR of galaxies, as shown in the Appendix.

Using the local overdensity of galaxies as a measure of the environment and a similar data set, Darvish et al. (2016) and Scoville et al. (2013) showed that the median SFR for the overall population of galaxies depends on the local environment out to $z \sim 1.1-1.2$ and is lower in denser regions. We checked this using our current sample of galaxies and found that our results based on the local overdensity of galaxies are in agreement with previous studies (Scoville et al. 2013; Darvish et al. 2016). However, as we showed in Figure 9, the median SFR for the general galaxy population depends on the global cosmic web only to $z \lesssim 0.8$. This might suggest that the local environment of galaxies is more fundamental than the global cosmic web environment, at least in this redshift range $(0.8 \lesssim$ $z \lesssim 1.2$ ).

Peng et al. (2012) in the local universe and Kovač et al. (2014) out to $z \sim 0.7$ showed that satellite quenching is the main driver of the environmental effects. As we showed in Figures 9-11, the observed trends for satellites resemble those of the overall galaxy population, suggesting the dominant role of satellite galaxies in shaping the general environmental trends, in agreement with (Peng et al. 2012; Kovač et al. 2014). At a given stellar mass, satellite galaxies are more likely to be on the quenched side of the sSFR distribution, have a greater probability of being red, and have a larger fraction of quenched systems than centrals (see, e.g., Zehavi et al. 2011; Wetzel et al. 2012, 2013; Knobel et al. 2013; Hirschmann et al. 2014; Omand et al. 2014, also Figure 11), which explains why they seem to dominate the environmental trends.

For SF galaxies, the cosmic web independence of the median SFR at $z \gtrsim 0.5$ is consistent with Darvish et al. (2014, 2015a). Darvish et al. (2014) showed that for a $z \sim 0.83 \mathrm{LSS}$, the observed median SFR for $\mathrm{H} \alpha$ SF galaxies is almost independent of the cosmic web. Darvish et al. (2015b) showed that the equivalent width of [O II] line (a measure of the sSFR) as a function of stellar mass is almost the same for filament and field SF galaxies at $z \sim 0.53$. Furthermore, Erfanianfar et al. (2016) found that the main-sequence of SF galaxies at $0.5<z<1.1$ is similar for group, filament-like, and field galaxies but at $0.15<z<1.1$, they found that group galaxies deviate from the main sequence toward lower SFRs at a fixed stellar mass. These are fully consistent with our results for the $\mathrm{SF}$ galaxies.

However, as already discussed in Darvish et al. (2016), for SF galaxies, there is no consistency on this topic in the literature over different redshifts, as some studies have found an environmental dependence of SFR for SF galaxies (e.g., von der Linden et al. 2010; Vulcani et al. 2010; Patel et al. 2011; Haines et al. 2013; Tran et al. 2015; Erfanianfar et al. 2016), 

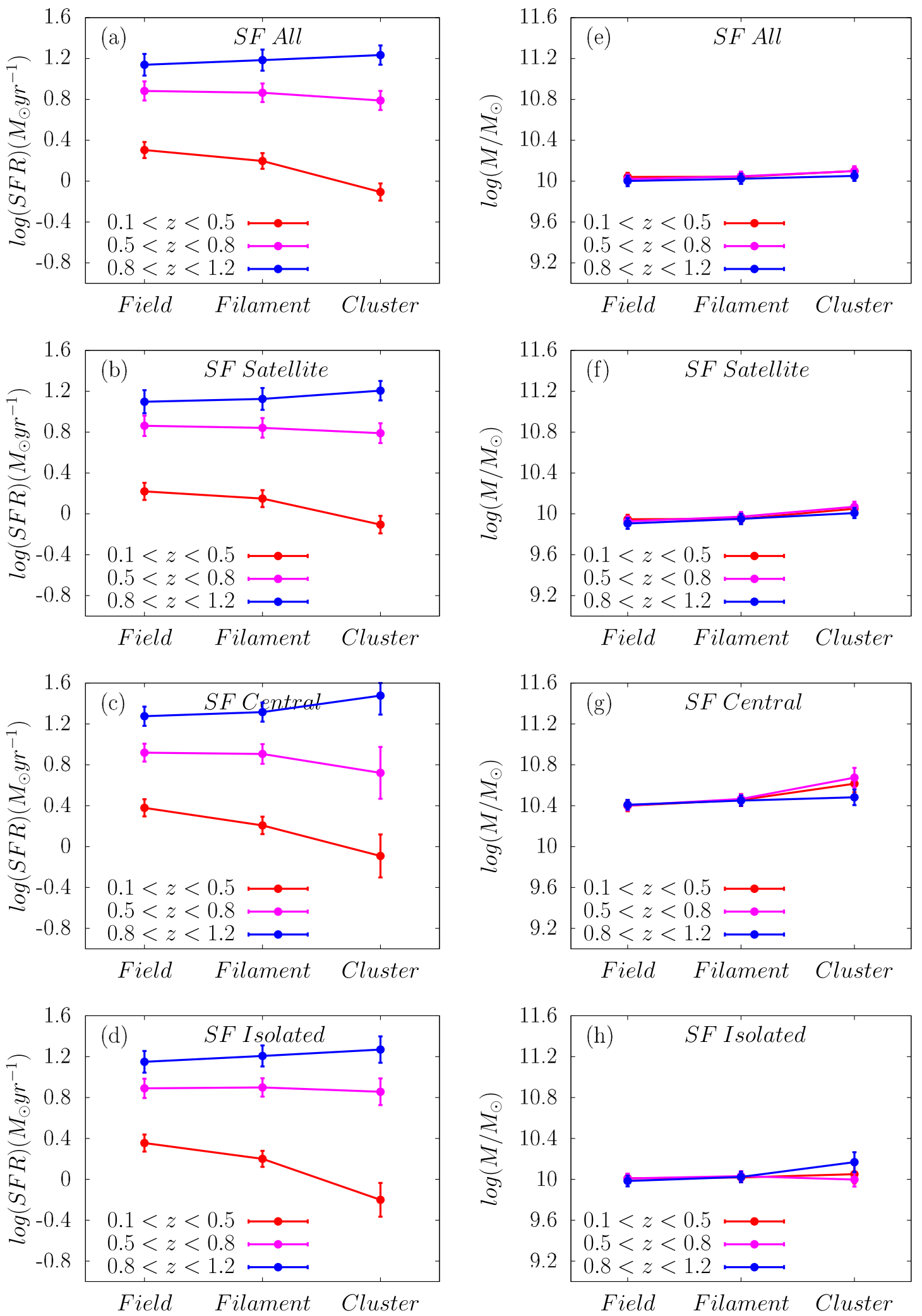

Figure 10. (a)-(d) Median SFR for all star-forming, satellite star-forming, central star-forming, and isolated star-forming galaxies in the cosmic web, respectively. At $z \gtrsim 0.5$, and within the uncertainties, the median SFR for star-forming centrals, satellites, isolated, and all do not much depend on the cosmic web. However, at $z \lesssim 0.5$, all satellite, central, and isolated (and all) star-forming galaxies show a $\sim 0.3-0.4$ dex decline in their median SFR. These results have implications for the nature of galaxy quenching in the cosmic web and their qualitative timescales (Section 4). (e)-(h) Median stellar mass for all star-forming, satellite star-forming, central star-forming, and isolated star-forming galaxies in the cosmic web, respectively. Within the uncertainties, we see almost no change or a slight increase in some cases ( 0.2-0.3 dex in maximum) in the median stellar mass of star-forming galaxies from the field to clusters. Therefore, stellar mass differences in different parts of the cosmic web cannot much explain the observed cosmic web dependence of the SFRs here or make the trends even stronger. 

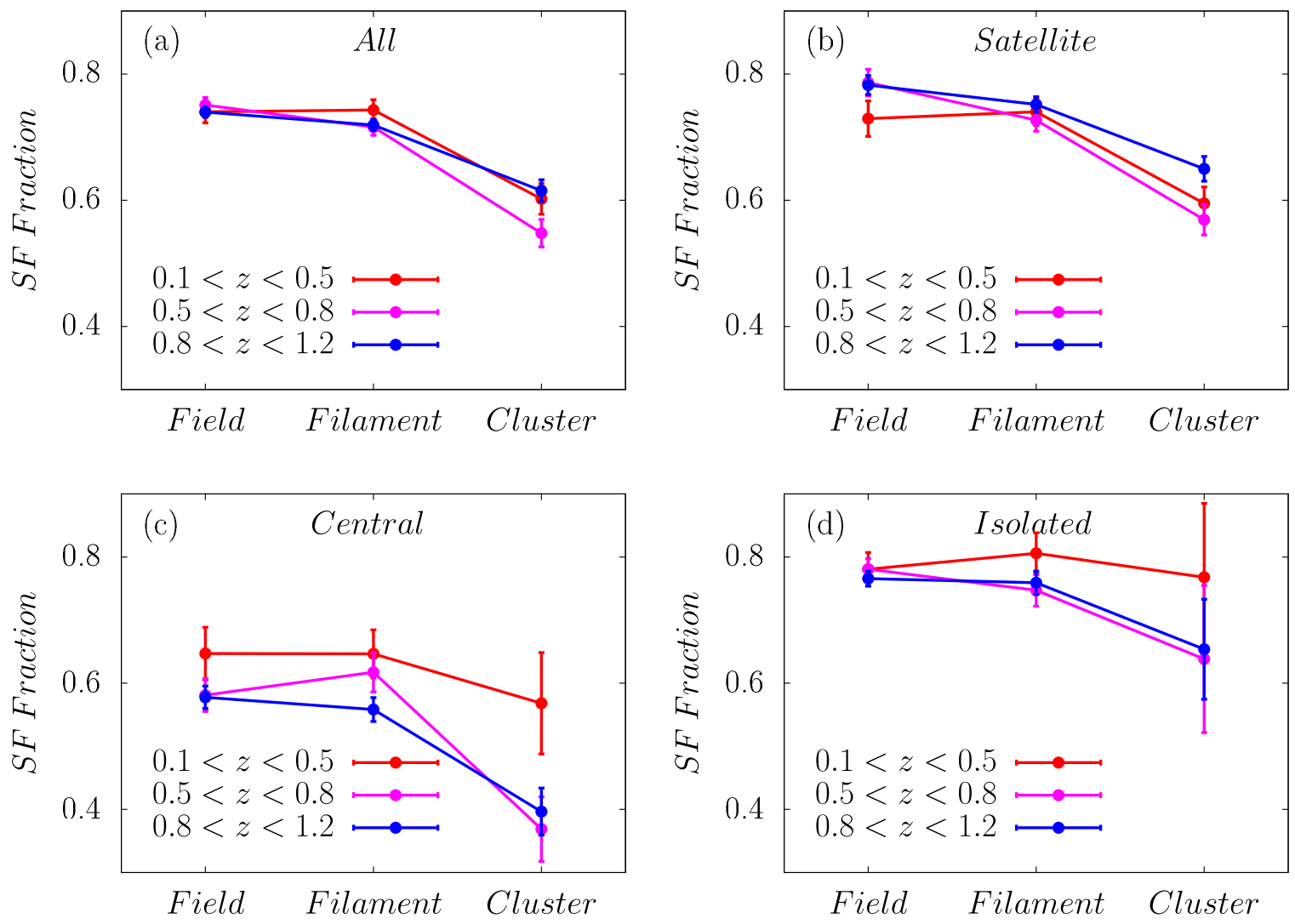

Figure 11. (a)-(d) Star-forming (SF) fraction for all, satellite, central, and isolated galaxies in the cosmic web, respectively. The SF fraction declines from the field to clusters for the overall population of galaxies and for satellite systems at all the redshifts considered in this work, without any significant redshift evolution. At $z \lesssim 0.5$ and within the uncertainties, the SF fraction is similar in different environments for central and isolated systems and it declines from the field to clusters at $z \gtrsim 0.5$ for them. Given the results in this figure and Figures 9 and 10, we conclude that most satellites experience a fast quenching mechanism as they fall from the field into clusters through filaments, whereas central and isolated galaxies mostly undergo a fast environmental quenching process at $z \gtrsim 0.5$ and a slow mechanism at $z \lesssim 0.5$. Note that the difference between filament and field systems is not significant, within the uncertainties.

whereas others found none/weak (e.g., Patel et al. 2009; Peng et al. 2010; Muzzin et al. 2012; Wijesinghe et al. 2012; Koyama et al. 2013; Cen 2014; Darvish et al. 2014; Lin et al. 2014; Ricciardelli et al. 2014; Vogelsberger et al. 2014; Darvish et al. 2015a; Stroe et al. 2015; Vulcani et al. 2016b).

For example, even at $0.1 \leqslant z<0.5$, Darvish et al. (2016) found that the median SFR for SF galaxies, even at fixed stellar mass, is independent of the local overdensities within the uncertainties. However, using a similar data set and redshift range in this work $(0.1 \leqslant z \leqslant 0.5)$, we see that satellite, central, and isolated SF galaxies show a decline in their median SFR in clusters compared to the field. Part of the difference might be due to this idea that the global cluster membership (and the global halo properties) might be more important than the local overdensities in determining the star formation activity of SF galaxies at lower redshifts. Another possibility is that dividing the galaxies into different overdensity bins results in a small sample size in each bin, which might wash out any global environmental dependence of SFR for SF galaxies at lower redshifts, particularly when combined with typically large SED-based SFR uncertainties.

Several studies have seen an enhancement in the fraction of active SF galaxies in filaments with respect to clusters and the field, likely due to interaction between galaxies as they fall into denser regions of clusters along the filaments (see, e.g., Fadda et al. 2008; Darvish et al. 2014). We do not see a significant enhancement in the SF fraction in filaments for our sample (see Figure 11). Part of this discrepancy might be due to the different type of SF galaxies with different star formation timescales used. For example, Darvish et al. (2014) used $\mathrm{H} \alpha$ emitters with much shorter star formation timescales than our current study (which relies on SED template fitting SFRs) and Fadda et al. (2008) used starburst galaxies in their study. In other words, if the star formation activity really enhances in filaments in very short timescales, shorter than the SED-based SFRs, we will not be able to see that in this study.

Sobral et al. (2016) showed that $\mathrm{H} \alpha$ SF galaxies at $z \sim 0.4$ are significantly dustier than their field counterparts, resulting in apparently lower SFRs in denser environment if dust correction has not been applied in SFR estimation. We note that our SED-based SFR has taken the dust correction into account and in a statistical sense, cannot significantly alter our results here.

In the local universe, Poudel et al. (2017) recently found that at fixed group mass and large-scale luminosity density, central galaxies in filaments have lower sSFR than those outside of filaments. Our results at $0.1<z<0.5$ for central galaxies (e.g., Figure 9) show a similar trend in filaments compared to those in the field, consistent with Poudel et al. (2017).

By combining the SDSS data with a high-resolution $N$-body simulation, Wetzel et al. (2013) showed that for satellites of log $\left(M / M_{\odot}\right)>9.7$ at $z \sim 0$, SFRs evolve unaffected for 2-4 Gyr after infall into a halo, after which star formation quenches rapidly, with an e-folding timescale of $0.2-0.8 \mathrm{Gyr}$ and shorter quenching timescales for more massive satellites. Recently, Hahn et al. (2016) extended this to central galaxies, showing 
that it takes a total migration time of $\sim 4 \mathrm{Gyr}$ from mainsequence to quiescence for $\log \left(M / M_{\odot}\right)=10.5$ central galaxies, $\sim 2 \mathrm{Gyr}$ longer than satellites (also see Tal et al. 2014). These studies are qualitatively consistent with our results, suggesting a slower quenching timescale for the majority of centrals at $z \lesssim 0.5$, possibly due to a slow quenching process such as strangulation. However, for the majority of satellites, a fast quenching mechanism after their infall into their host cluster halos, such as ram pressure stripping, can better explain our results. We highlight that our results also support a fast environmental quenching mechanism for both centrals and satellites at $z \gtrsim 0.5$. We also note that due to the slow nature of the strangulation mechanism, it makes sense that we only see its effects on the evolution of galaxies only at lower redshifts (e.g., Figure 10).

The stellar mass difference between satellites and centrals and their nature might explain why most satellites seem to be undergoing ram pressure stripping (with its fast quenching timescale) but centrals and isolated galaxies do not, at least at $z \lesssim 0.5$. By definition, we select centrals as the most massive system in each group, which automatically induces a mass difference relative to satellites (e.g., compare Figures 9 (f) and (g)). The typically lower mass of satellites results in a weaker binding energy of the galactic system as it experiences ram pressure stripping. Moreover, ram pressure stripping depends on the square of the relative speed between the IGM of the dense environment and the motion of the galaxy within it. Centrals are almost localized to the center of the gravitational potential of their group, whereas satellites typically move around in the system, which automatically leads to a more effective gas stripping for them. If isolated galaxies are centrals with faint satellites, therefore, in principle, they should behave like centrals. If they are ejected satellites, they are orbiting beyond their halo's effective radius and therefore, are not much affected by the ram pressure stripping. However, we note that determining the exact physical mechanisms for quenching of these systems and the quantitative timescales require a more careful analysis, which is beyond the scope of this work.

It is worth noting that despite the large size of the sample used in this study, the overall uncertainties are still large. This sets the need for extremely large-volume surveys in the near future, such as LSST, Euclid, and WFIRST, to substantially tackle this issue.

\section{Summary and Conclusion}

We use a mass complete $\left(\log \left(M / M_{\odot}\right) \geqslant 9.6\right)$ sample of galaxies in the $\sim 2 \mathrm{deg}^{2}$ COSMOS field out to $z=1.2$ to construct the density field from which the comic web of galaxies is extracted. Using the density field Hessian matrix, we disentangle the cosmic web into clusters, filaments, and the general field. We provide a catalog of environmental measures such as the local density, cosmic web, and central, satellite, and isolated dichotomy to the community. We compare the basic properties of the cosmic web with simulations and investigate the star formation activity of galaxies in the cosmic web to $z=1.2$ with the following main results.

1. The general properties of our estimated cosmic web, such as the volume filling fraction of different components, are in an overall good agreement with numerical simulations that use the same classification algorithm.
2. Within the uncertainties, we do not find a significant difference between the star formation activity in filaments and the field.

3. The median SFR of the overall population of galaxies declines in the cosmic web from the field to clusters at $z \lesssim 0.8$ and flattens out at higher redshifts. Satellite galaxies experience the largest decline of $\sim 1 \mathrm{dex}$ especially at lower redshifts, whereas centrals and isolated galaxies show a decline of $\sim 0.4-0.5 \mathrm{dex}$ in the same redshift range.

4. The median SFR of the SF galaxies in the cosmic web declines by $\sim 0.3-0.4$ dex from the field to clusters for satellites, centrals, and isolated systems at $z \lesssim 0.5$ and is almost independent of the comic web at higher redshifts.

5. The SF fraction for the overall galaxy population and satellite systems declines from the field to clusters at all the redshifts considered in this work, without any significant redshift evolution. For central galaxies, the $\mathrm{SF}$ fraction is almost unchanged in the cosmic web at $z \lesssim 0.5$ and it declines from the field to clusters at $z \gtrsim 0.5$.

6. The decline in the median SFR for satellite galaxies in the cosmic web is mainly due to a decrease in the fraction of satellite SF galaxies from the field to clusters, suggesting a rapid quenching mechanism for the majority of satellites in the web. For central galaxies, the slighter decline in the median SFR at $z \lesssim 0.5$ is mainly attributed to an overall decline in the SFR of individual central galaxies, suggesting a slower quenching process for central systems at $z \lesssim 0.5$. However, centrals at $z \gtrsim 0.5$ should also undergo a fast environmental quenching mechanism.

This paper is the first one in a series studying the explicit role of the cosmic web on galaxy properties over the past $\sim 8 \mathrm{Gyr}$. In a following paper, we will investigate the dependence of other galaxy properties on the cosmic web and will explain the results in the context of galaxy formation and evolution.

We are immensely grateful to the anonymous referee for their useful comments that greatly improved the quality of this work. B.D. acknowledges financial support from NASA through the Astrophysics Data Analysis Program (ADAP), grant number NNX12AE20G. B.D. wishes to thank Adam Muzzin for his useful comments. D.S. acknowledges financial support from the Netherlands Organisation for Scientific Research (NWO) through a Veni fellowship and from Lancaster University through an Early Career Internal Grant A100679. This work is based on data products from observations made with ESO Telescopes at the La Silla Paranal Observatory under ESO programme ID 179.A-2005 and on data products produced by TERAPIX and the Cambridge Astronomy Survey Unit on behalf of the UltraVISTA consortium.

\section{Appendix}

In Section 4.2, we investigated the relation between the cosmic web and the median SFR of the overall galaxy population, satellites, centrals, and the isolated systems. Here, we investigate similar trends between the median sSFR of different galaxy types as a function of cosmic web 


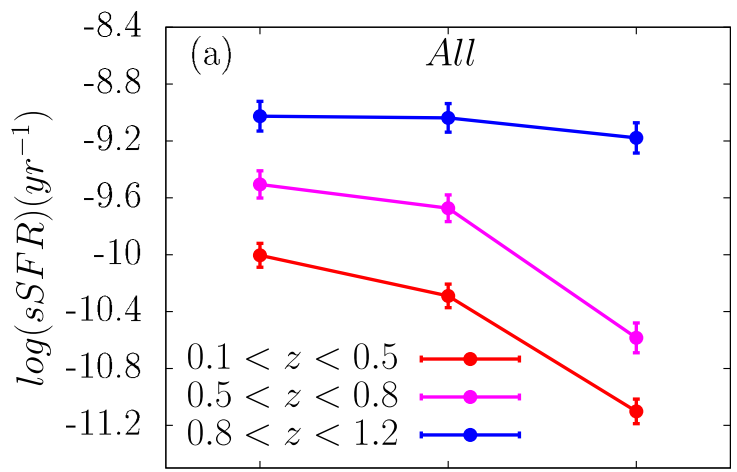

Field Filament Cluster

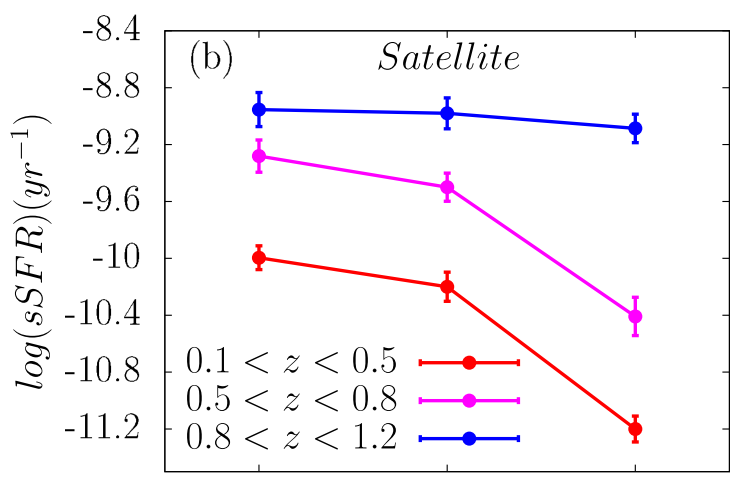

Field Filament Cluster
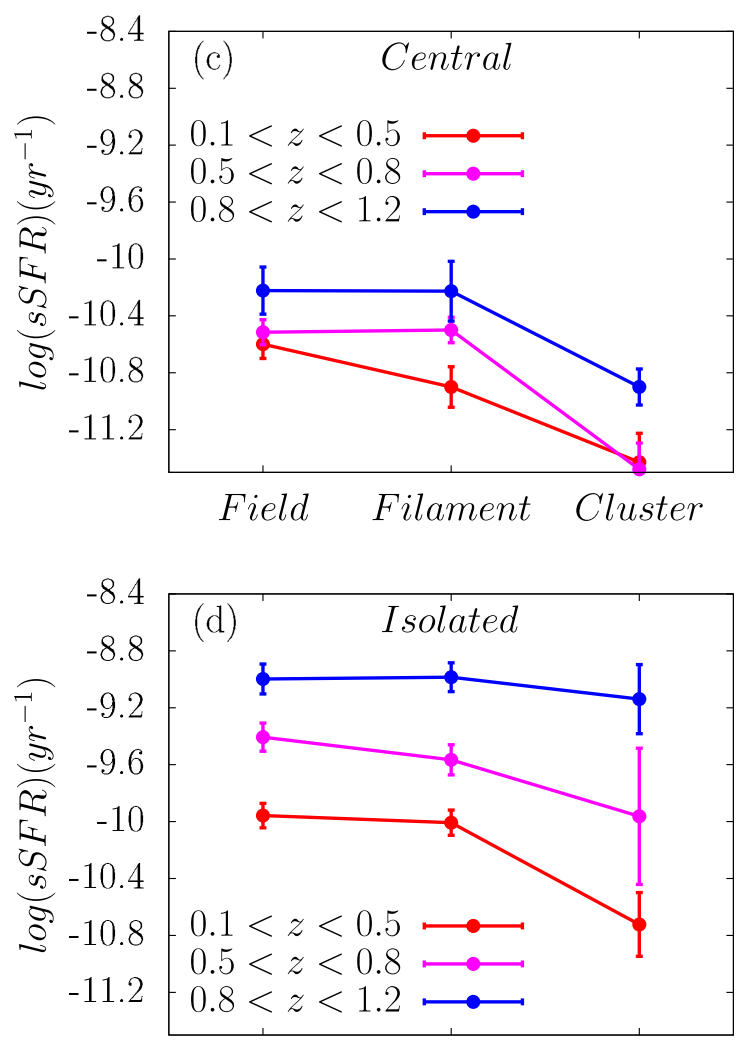

Field Filament Cluster

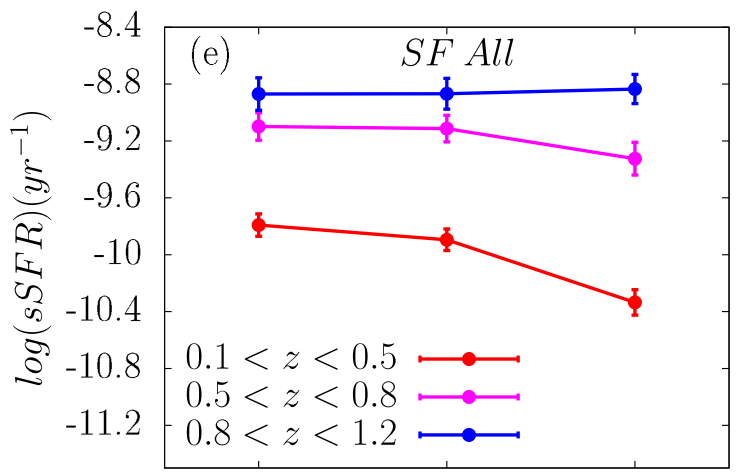

Field Filament Cluster

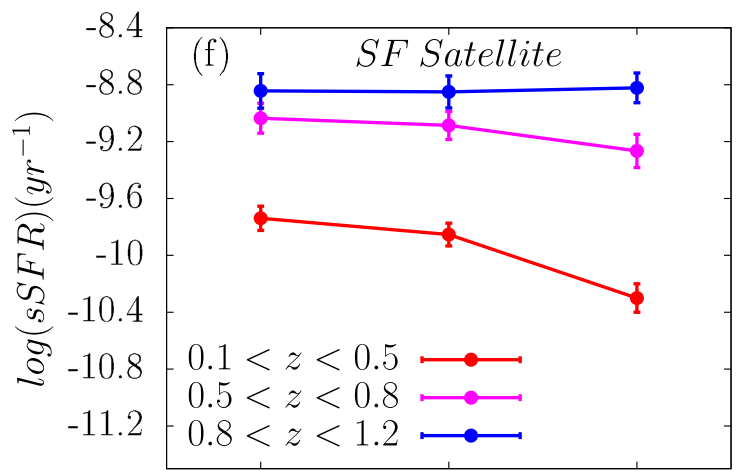

Field Filament Cluster

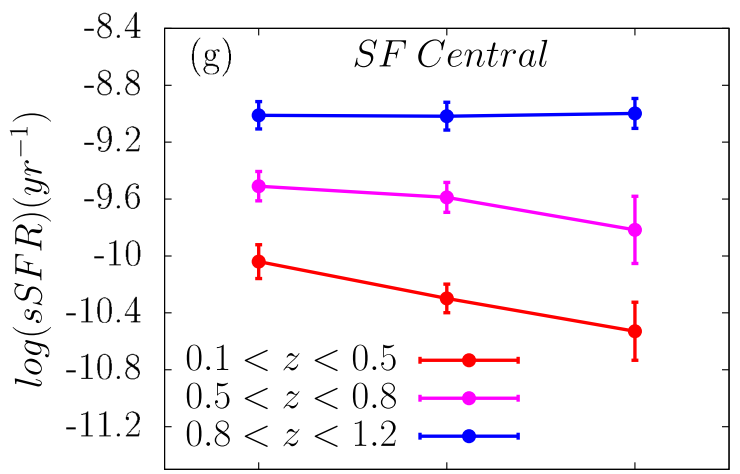

Field Filament Cluster

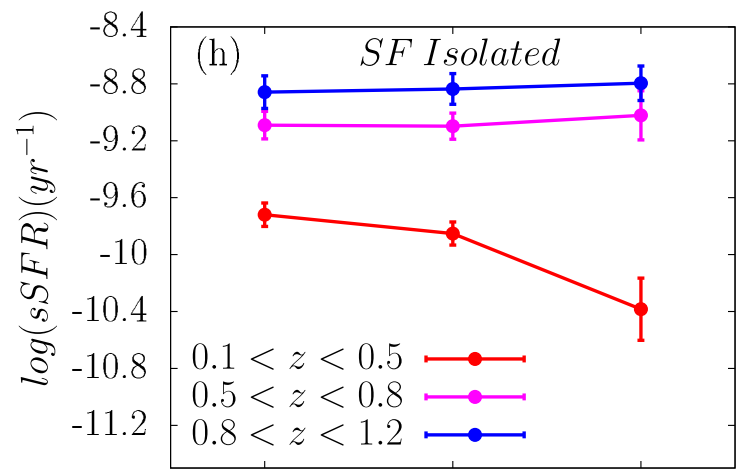

Field Filament Cluster

Figure 12. (a)-(d) Similar to Figures 9(a)-(d), but now with the median sSFR as the quantity under investigation. (e)-(h) Similar to Figures 10(a)-(d), but now with the median SSFR as the quantity under investigation. 
environments, with results similar to those in Section 4.2, as shown in Figure 12.

\section{References}

Abadi, M. G., Moore, B., \& Bower, R. G. 1999, MNRAS, 308, 947 Alonso, D., Hadzhiyska, B., \& Strauss, M. A. 2016, MNRAS, 460, 256 Alpaslan, M., Driver, S., Robotham, A. S. G., et al. 2015, MNRAS, 451, 3249 Alpaslan, M., Grootes, M., Marcum, P. M., et al. 2016, MNRAS, 457, 2287 Alpaslan, M., Robotham, A. S. G., Driver, S., et al. 2014a, MNRAS, 438, 177 Alpaslan, M., Robotham, A. S. G., Obreschkow, D., et al. 2014b, MNRAS, 440, L106

Altay, G., Colberg, J. M., \& Croft, R. A. C. 2006, MNRAS, 370, 1422

Aragón-Calvo, M. A., Jones, B. J. T., van de Weygaert, R., \& van der Hulst, J. M. 2007a, A\&A, 474, 315

Aragón-Calvo, M. A., Neyrinck, M. C., \& Silk, J. 2016, MNRAS, submitted (arXiv:1607.07881)

Aragón-Calvo, M. A., Platen, E., van de Weygaert, R., \& Szalay, A. S. 2010a, ApJ, 723, 364

Aragón-Calvo, M. A., van de Weygaert, R., \& Jones, B. J. T. 2010b, MNRAS, 408, 2163

Aragón-Calvo, M. A., van de Weygaert, R., Jones, B. J. T., \& van der Hulst, J. M. 2007b, ApJL, 655, L5

Aragón-Calvo, M. A., Weygaert, R. v. d., Jones, B. J. T., \& Mobasher, B. 2015, MNRAS, 454, 463

Balogh, M. L., Navarro, J. F., \& Morris, S. L. 2000, ApJ, 540, 113

Becker, G. D., Bolton, J. S., \& Lidz, A. 2015, PASA, 32, e045

Berti, A. M., Coil, A. L., Behroozi, P. S., et al. 2017, ApJ, 834, 87

Beygu, B., Kreckel, K., van der Hulst, J. M., et al. 2016, MNRAS, 458, 394

Biviano, A., Fadda, D., Durret, F., Edwards, L. O. V., \& Marleau, F. 2011, A\&A, 532, A77

Bond, J. R., Kofman, L., \& Pogosyan, D. 1996, Natur, 380, 603

Bond, N. A., Strauss, M. A., \& Cen, R. 2010, MNRAS, 409, 156

Briel, U. G., \& Henry, J. P. 1995, A\&A, 302, L9

Brouwer, M. M., Cacciato, M., Dvornik, A., et al. 2016, MNRAS, 462, 4451 Bruzual, G., \& Charlot, S. 2003, MNRAS, 344, 1000

Cantalupo, S., Arrigoni-Battaia, F., Prochaska, J. X., Hennawi, J. F., \& Madau, P. 2014, Natur, 506, 63

Capak, P., Aussel, H., Ajiki, M., et al. 2007, ApJS, 172, 99

Cautun, M., van de Weygaert, R., \& Jones, B. J. T. 2013, MNRAS, 429, 1286

Cautun, M., van de Weygaert, R., Jones, B. J. T., \& Frenk, C. S. 2014, MNRAS, 441, 2923

Cen, R. 2014, ApJ, 781, 38

Cen, R., \& Ostriker, J. P. 1999, ApJ, 514, 1

Chabrier, G. 2003, PASP, 115, 763

Chen, S., Wang, H., Mo, H. J., \& Shi, J. 2016a, ApJ, 825, 49

Chen, Y.-C., Ho, S., Mandelbaum, R., et al. 2016b, MNRAS, 466, 1880

Chen, Y.-C., Ho, S., Tenneti, A., et al. 2015, MNRAS, 454, 3341

Codis, S., Pichon, C., Devriendt, J., et al. 2012, MNRAS, 427, 3320

Codis, S., Pichon, C., \& Pogosyan, D. 2015, MNRAS, 452, 3369

Colberg, J. M. 2007, MNRAS, 375, 337

Colless, M., Peterson, B. A., Jackson, C., et al. 2003, arXiv:astro-ph/0306581

Cooper, M. C., Newman, J. A., Madgwick, D. S., et al. 2005, ApJ, 634, 833

Darvish, B., Mobasher, B., Sobral, D., et al. 2015a, ApJ, 814, 84

Darvish, B., Mobasher, B., Sobral, D., et al. 2016, ApJ, 825, 113

Darvish, B., Mobasher, B., Sobral, D., Scoville, N., \& Aragon-Calvo, M. 2015b, ApJ, 805, 121

Darvish, B., Sobral, D., Mobasher, B., et al. 2014, ApJ, 796, 51

Davé, R., Cen, R., Ostriker, J. P., et al. 2001, ApJ, 552, 473

Davis, M., Efstathiou, G., Frenk, C. S., \& White, S. D. M. 1985, ApJ, 292, 371

Dekel, A., Birnboim, Y., Engel, G., et al. 2009, Natur, 457, 451

Doroshkevich, A., Tucker, D. L., Allam, S., \& Way, M. J. 2004, A\&A, 418, 7

Duarte, M., \& Mamon, G. A. 2014, MNRAS, 440, 1763

Dubois, Y., Pichon, C., Welker, C., et al. 2014, MNRAS, 444, 1453

Eardley, E., Peacock, J. A., McNaught-Roberts, T., et al. 2015, MNRAS, 448, 3665

Erfanianfar, G., Popesso, P., Finoguenov, A., et al. 2016, MNRAS, 455, 2839 Fabian, A. C. 2012, ARA\&A, 50, 455

Fadda, D., Biviano, A., Marleau, F. R., Storrie-Lombardi, L. J., \& Durret, F. 2008, ApJL, 672, L9

Falck, B. L., Neyrinck, M. C., \& Szalay, A. S. 2012, ApJ, 754, 126

Farouki, R., \& Shapiro, S. L. 1981, ApJ, 243, 32

Filho, M. E., Sánchez Almeida, J., Muñoz-Tuñón, C., et al. 2015, ApJ, 802, 82

Finoguenov, A., Guzzo, L., Hasinger, G., et al. 2007, ApJS, 172, 182
Forero-Romero, J. E., Hoffman, Y., Gottlöber, S., Klypin, A., \& Yepes, G. 2009, MNRAS, 396, 1815

Frangi, A. F., Niessen, W. J., Vincken, K. L., \& Viergever, M. A. 1998, LNCS, 1496, 130

Geller, M. J., \& Huchra, J. P. 1989, Sci, 246, 897

George, M. R., Leauthaud, A., Bundy, K., et al. 2011, ApJ, 742, 125

González, R. E., \& Padilla, N. D. 2010, MNRAS, 407, 1449

Gonzalez, R. E., Prieto, J., Padilla, N., \& Jimenez, R. 2016, MNRAS, 464, 4666

Gunn, J. E., \& Gott, J. R., III 1972, ApJ, 176, 1

Guo, Q., Tempel, E., \& Libeskind, N. I. 2015, ApJ, 800, 112

Hahn, C., Tinker, J. L., \& Wetzel, A. R. 2016, arXiv:1609.04398

Hahn, O., Carollo, C. M., Porciani, C., \& Dekel, A. 2007a, MNRAS, 381, 41

Hahn, O., Porciani, C., Carollo, C. M., \& Dekel, A. 2007b, MNRAS, 375, 489

Haider, M., Steinhauser, D., Vogelsberger, M., et al. 2016, MNRAS, 457, 3024

Haines, C. P., Pereira, M. J., Smith, G. P., et al. 2013, ApJ, 775, 126

Hartley, W. G., Conselice, C. J., Mortlock, A., Foucaud, S., \& Simpson, C. 2015, MNRAS, 451, 1613

Hatfield, P. W., \& Jarvis, M. J. 2016, MNRAS, submitted (arXiv:1606.08989)

Hearin, A. P., Watson, D. F., \& van den Bosch, F. C. 2015, MNRAS, 452, 1958

Hirschmann, M., De Lucia, G., Wilman, D., et al. 2014, MNRAS, 444, 2938

Hoffman, Y., Metuki, O., Yepes, G., et al. 2012, MNRAS, 425, 2049

Hopkins, P. F., Kereš, D., Oñorbe, J., et al. 2014, MNRAS, 445, 581

Huchra, J. P., \& Geller, M. J. 1982, ApJ, 257, 423

Ilbert, O., Arnouts, S., Le Floc'h, E., et al. 2015, A\&A, 579, A2

Ilbert, O., Capak, P., Salvato, M., et al. 2009, ApJ, 690, 1236

Ilbert, O., McCracken, H. J., Le Fèvre, O., et al. 2013, A\&A, 556, A55

Jarrett, T. 2004, PASA, 21, 396

Jasche, J., Kitaura, F. S., Li, C., \& Enßlin, T. A. 2010, MNRAS, 409, 355

Joachimi, B., Cacciato, M., Kitching, T. D., et al. 2015, SSRv, 193, 1

Jones, B. J. T., van de Weygaert, R., \& Aragón-Calvo, M. A. 2010, MNRAS, 408, 897

Jones, D. H., Read, M. A., Saunders, W., et al. 2009, MNRAS, 399, 683

Kang, X., \& Wang, P. 2015, ApJ, 813, 6

Kashikawa, N., \& Okamura, S. 1992, PASJ, 44, 493

Kauffmann, G., Li, C., Zhang, W., \& Weinmann, S. 2013, MNRAS, 430, 1447

Kawinwanichakij, L., Quadri, R. F., Papovich, C., et al. 2016, ApJ, 817, 9

Kereš, D., Katz, N., Weinberg, D. H., \& Davé, R. 2005, MNRAS, 363, 2

Kiessling, A., Cacciato, M., Joachimi, B., et al. 2015, SSRv, 193, 67

Knobel, C., Lilly, S. J., Kovač, K., et al. 2013, ApJ, 769, 24

Kovač, K., Lilly, S. J., Knobel, C., et al. 2014, MNRAS, 438, 717

Koyama, Y., Smail, I., Kurk, J., et al. 2013, MNRAS, 434, 423

Lai, C.-C., Lin, L., Jian, H.-Y., et al. 2016, ApJ, 825, 40

Laigle, C., McCracken, H. J., Ilbert, O., et al. 2016, ApJS, 224, 24

Larson, R. B., Tinsley, B. M., \& Caldwell, C. N. 1980, ApJ, 237, 692

Leclercq, F., Jasche, J., \& Wandelt, B. 2015, JCAP, 6, 015

Lee, J., \& Erdogdu, P. 2007, ApJ, 671, 1248

Lee, N., Sanders, D. B., Casey, C. M., et al. 2013, ApJ, 778, 131

Lee, N., Sanders, D. B., Casey, C. M., et al. 2015, ApJ, 801, 80

Libeskind, N. I., Hoffman, Y., Forero-Romero, J., et al. 2013, MNRAS, 428, 2489

Lilly, S. J., Le Brun, V., Maier, C., et al. 2009, ApJS, 184, 218

Lin, L., Jian, H.-Y., Foucaud, S., et al. 2014, ApJ, 782, 33

Malavasi, N., Pozzetti, L., Cucciati, O., Bardelli, S., \& Cimatti, A. 2016, A\&A, 585, A116

Martin, D. C., Chang, D., Matuszewski, M., et al. 2014a, ApJ, 786, 106

Martin, D. C., Chang, D., Matuszewski, M., et al. 2014b, ApJ, 786, 107

Martin, D. C., Matuszewski, M., Morrissey, P., et al. 2015, Natur, 524, 192

Martin, D. C., Matuszewski, M., Morrissey, P., et al. 2016, ApJL, 824, L5

McCracken, H. J., Milvang-Jensen, B., Dunlop, J., et al. 2012, A\&A, 544, A156

Merritt, D. 1983, ApJ, 264, 24

Moore, B., Lake, G., \& Katz, N. 1998, ApJ, 495, 139

Moster, B. P., Somerville, R. S., Newman, J. A., \& Rix, H.-W. 2011, ApJ, 731,113

Muzzin, A., Wilson, G., Yee, H. K. C., et al. 2012, ApJ, 746, 188

Navarro, J. F., Abadi, M. G., \& Steinmetz, M. 2004, ApJL, 613, L41

Nicastro, F., Mathur, S., Elvis, M., et al. 2005, Natur, 433, 495

Novikov, D., Colombi, S., \& Doré, O. 2006, MNRAS, 366, 1201

Omand, C. M. B., Balogh, M. L., \& Poggianti, B. M. 2014, MNRAS, 440, 843

Pandey, B., \& Sarkar, S. 2016, MNRAS, 467, L6

Patel, S. G., Holden, B. P., Kelson, D. D., Illingworth, G. D., \& Franx, M. 2009, ApJL, 705, L67

Patel, S. G., Kelson, D. D., Holden, B. P., Franx, M., \& Illingworth, G. D. 2011, ApJ, 735, 53 
Paz, D. J., Stasyszyn, F., \& Padilla, N. D. 2008, MNRAS, 389, 1127

Peebles, P. J. E. 1969, ApJ, 155, 393

Peng, Y.-j., Lilly, S. J., Kovač, K., et al. 2010, ApJ, 721, 193

Peng, Y.-j., Lilly, S. J., Renzini, A., \& Carollo, M. 2012, ApJ, 757, 4

Penny, S. J., Brown, M. J. I., Pimbblet, K. A., et al. 2015, MNRAS, 453, 3519

Pimbblet, K. A. 2005, MNRAS, 358, 256

Porter, S. C., Raychaudhury, S., Pimbblet, K. A., \& Drinkwater, M. J. 2008, MNRAS, 388, 1152

Poudel, A., Heinämäki, P., Tempel, E., et al. 2014, 597, A86

Pozzetti, L., Bolzonella, M., Zucca, E., et al. 2010, A\&A, 523, A13

Quadri, R. F., Williams, R. J., Franx, M., \& Hildebrandt, H. 2012, ApJ, 744, 88

Ricciardelli, E., Cava, A., Varela, J., \& Quilis, V. 2014, MNRAS, 445, 4045

Scharf, C., Donahue, M., Voit, G. M., Rosati, P., \& Postman, M. 2000, ApJL, 528, L73

Scoville, N., Arnouts, S., Aussel, H., et al. 2013, ApJS, 206, 3

Scoville, N., Aussel, H., Benson, A., et al. 2007a, ApJS, 172, 150

Scoville, N., Aussel, H., Brusa, M., et al. 2007b, ApJS, 172, 1

Shull, J. M., Smith, B. D., \& Danforth, C. W. 2012, ApJ, 759, 23

Smith, A. G., Hopkins, A. M., Hunstead, R. W., \& Pimbblet, K. A. 2012, MNRAS, 422, 25

Snedden, A., Coughlin, J., Phillips, L. A., Mathews, G., \& Suh, I.-S. 2016, MNRAS, 455, 2804

Snedden, A., Phillips, L. A., Mathews, G. J., et al. 2015, JCoPh, 299, 92

Sobral, D., Best, P. N., Smail, I., et al. 2011, MNRAS, 411, 675

Sobral, D., Stroe, A., Dawson, W. A., et al. 2015, MNRAS, 450, 630

Sobral, D., Stroe, A., Koyama, Y., et al. 2016, MNRAS, 458, 3443

Sousbie, T. 2011, MNRAS, 414, 350

Sousbie, T., Pichon, C., Colombi, S., Novikov, D., \& Pogosyan, D. 2008, MNRAS, 383, 1655

Stoica, R. S., Martínez, V. J., Mateu, J., \& Saar, E. 2005, A\&A, 434, 423

Stoica, R. S., Martínez, V. J., \& Saar, E. 2010, A\&A, 510, A38

Stroe, A., Sobral, D., Dawson, W., et al. 2015, MNRAS, 450, 646

Tal, T., Dekel, A., Oesch, P., et al. 2014, ApJ, 789, 164
Tempel, E., \& Libeskind, N. I. 2013, ApJL, 775, L42

Tempel, E., Stoica, R. S., Martínez, V. J., et al. 2014, MNRAS, 438, 3465

Tempel, E., Stoica, R. S., \& Saar, E. 2013, MNRAS, 428, 1827

Tran, K.-V. H., Nanayakkara, T., Yuan, T., et al. 2015, ApJ, 811, 28

Trowland, H. E., Lewis, G. F., \& Bland-Hawthorn, J. 2013, ApJ, 762, 72

Vogelsberger, M., Genel, S., Springel, V., et al. 2014, Natur, 509, 177

von der Linden, A., Wild, V., Kauffmann, G., White, S. D. M., \& Weinmann, S. 2010, MNRAS, 404, 1231

Vulcani, B., Poggianti, B. M., Finn, R. A., et al. 2010, ApJL, 710, L1

Vulcani, B., Treu, T., Nipoti, C., et al. 2016a, ApJ, submitted (arXiv:1610. 04615)

Vulcani, B., Treu, T., Schmidt, K. B., et al. 2016b, ApJ, 833, 178

Wang, H., Mo, H. J., Yang, X., et al. 2016, ApJ, 831, 164

Wang, H., Mo, H. J., Yang, X., \& van den Bosch, F. C. 2012, MNRAS, 420, 1809

Weinmann, S. M., van den Bosch, F. C., Yang, X., \& Mo, H. J. 2006, MNRAS, 366, 2

Welker, C., Dubois, Y., Pichon, C., Devriendt, J., \& Chisari, E. N. 2015, A\&A, submitted (arXiv:1512.00400)

Wetzel, A. R., Tinker, J. L., \& Conroy, C. 2012, MNRAS, 424, 232

Wetzel, A. R., Tinker, J. L., Conroy, C., \& van den Bosch, F. C. 2013, MNRAS, 432, 336

Wetzel, A. R., Tinker, J. L., Conroy, C., \& van den Bosch, F. C. 2014, MNRAS, 439, 2687

White, S. D. M. 1984, ApJ, 286, 38

Wijesinghe, D. B., Hopkins, A. M., Brough, S., et al. 2012, MNRAS, 423, 3679

Zappacosta, L., Mannucci, F., Maiolino, R., et al. 2002, A\&A, 394, 7

Zehavi, I., Zheng, Z., Weinberg, D. H., et al. 2011, ApJ, 736, 59

Zel'dovich, Y. B. 1970, A\&A, 5, 84

Zhang, Y., Yang, X., Faltenbacher, A., et al. 2009, ApJ, 706, 747

Zhang, Y., Yang, X., Wang, H., et al. 2013, ApJ, 779, 160 